Rev. Elev. Méd. vét. Pays trop., 1970, 23 (1) : 15-41

\title{
Notes sur les babesioses et l'anaplasmose des bovins à Madagascar III. Essais de traitement
}

\author{
par G. UILENBERG $\left(^{*}\right)$
}

\begin{abstract}
RESUME
L'auteur rapporte les résultats obtenus dans le traitement des babésioses et de l'anaplasmose bovines à Madagascar, en utilisant divers produits.
\end{abstract}

Les méthodes générales de travail ont été exposées auparavant (UILENBERG, 1968). Les essais de traitement ont été faits en grande partie sur des animaux splénectomisés, l'épreuve étant plus sévère que sur les animaux normaux, l'influence des produits sur les parasites étant moins masquée par les défenses de l'organisme.

Il faut distinguer entre les primo-infections transmises par la seringue, les primo-infections transmises par les tiques Boophilus microplus, (CANESTRINI, 1887), la première rechute suivant la splénectomie d'un porteur, et les rechutes secondaires, soit après primo-infection, soit après splénectomie d'un porteur.

a) Primo-infection transmise par la seringue.

Les essais de traitement sur Babesia bigemina (SMITH et KILBORNE, 1893) sont alors d'une grande valeur, la quasi-totalité des animaux succombant sans traitement; par contre, une proportion importante d'animaux infectés par la seringue avec Babesia argentina (LIGNIERES, 1909) et Anaplasma marginale (THEILER, 1910) guérit spontanément, encore plus ceux infectés avec Anaplasma centrale (THEILER, 1911). (Voir UILENBERG, 1969). Les essais de traitement sur ces trois

(*) Travail du Service d'Entomologie-Protozoologie, Laboratoire Central de l'Elevage, Tananarive; Institut d'Elevage et de Médecine Vétérinaire des Pays Tropicaux. Adresse actuelle: Centre de Recherches sur les Trypanosomiases Animales, Institut d'Elevage et de Médecine Vétérinaire des Pays Tropicaux, B.P. 39 , Bouar, République Centrafricaine. derniers parasites ne peuvent donc pas uniquement être jugés sur la guérison ou la mort et sont en fait assez peu concluants, sauf dans les cas où le traitement ne donne pas de résultat positif; le résultat d'un traitement effectué au début d'un accès a plus de valeur qu'après une parasitémie et hyperthermie ayant déjà duré plusieurs jours.

b) Primo-infection transmise par les tiques.

Les exemples suivants montreront que l'épreuve est beaucoup plus sévère que lors d'infection transmise par la seringue en ce qui concerne $B$. argentina, tandis que le mode de transmission ne semble pas avoir une grande influence en ce qui concerne le traitement de $B$. bigemina. Nous ignorons ce qui concerne les anaplasmes, étant donné que la transmission de l'anaplasmose par les tiques n'a été réussie qu'une seule fois jusqu ici à Madagascar (UILENBERG, sous presse). En définitive, pour expérimenter un produit sur $B$. argentina, il faudrait le faire sur animaux splénectomisés infectés par les tiques, infection que nous croyons être toujours ou presque toujours mortelle sans traitement.

c) Première rechute suivant la splénectomie d'un porteur chronique.

Les essais de traitement n'ont qu'une valeur limitée, étant donné qu'une proportion considérable des animaux guérit spontanément des quatre infections en question (UILENBERG, 1969). Aussi nous ne donnerons le plus souvent aucun détail sur ces essais. 


\section{d) Rechutes secondaires.}

La majorité des animaux guérit spontanément des rechutes à $B$, argentina et aux anaplasmes, et un traitement est également superflu pour une grande proportion des sujets faisant des rechutes à $B$. bigemina. Aussi les traitements de telles rechutes ne seront généralement pas rapportés.

Il ne nous semble pas qu'il faille tenir compte d'éventuelles rechutes sévères après traitement de splénectomisés, bien que nous les rapportions parfois. En effet, l'installation de l'état de prémunition sur les splénectomisés est, au début, beaucoup plus lente et moins efficace que sur les animaux normaux.

Quelques essais de traitement ont également pu être faits sur des animaux normaux. Dans ce cas l'épreuve est moins sévère pour chacun des quatre parasites en question, mais il faut tenir compte de l'âge (les jeunes étant moins sensibles), et de la race (en particulier en ce qui concerne $B$. argentina), (UILENBERG, 1968).

On a jugé de l'efficacité des traitements d'après la disparition des parasites du sang périphérique, les réactions thermiques et les signes cliniques, éventuellement d'après la dégénérescence des parasites. Nous signalerons, lorsqu'il a été possible de le déterminer, si le traitement a entraîné une stérilisation de l'organisme ou non. Cette stérilisation constitue un désavantage dans les régions enzootiques (comme Madagascar dans sa totalité) où il est préférable que tous les animaux restent prémunis, mais elle peut être un avantage dans les régions marginales où il peut être souhaitable d'éliminer les réservoirs d'infection.

Le degré de parasitémie est exprimé par des signes subjectifs: $(+),+,++$ et +++ . Bien que divers auteurs donnent le pourcentage d'érythrocytes infestés (souvent au dixième de degré près !), nous avons trouvé un comptage quotidien sur plusieurs frottis impraticable; la distribution des parasites sur le frottis est déjà tellement irrégulière qu'aucune méthode de comptage essayée ne nous a donné des résultats constants; de plus, le nombre d'érythrocytes par champ varie également d'un point à l'autre sur un même frottis, et d'un frottis à l'autre. (Voir aussi GARNHAM, 1966, p. 1040) et BECKMAN et SMITH, 1943.)

Pour ces raisons nous nous limitons aux signes subjectifs que nous pensons être suffisamment précis dans un but comparatif.

Signification des signes:

$(+)$ Parasitémie très faible à assez faible.

+ Parasitémie assez importante.

++ Parasitémie importante.

+++ Parasitémie très importante.

(La valeur de chaque signe n'est évidemment pas la même en pourcentage d'érythrocytes infestés pour chaque parasite différent; par exemple $\boldsymbol{B}$. bigemina atteint une parasitémie beaucoup plus élevée que $B$. argentina.)

Nous n'avons pas pu expérimenter tous les produits réputés d'avoir une action sur les parasites qui nous concernent; nous nous sommes limités à ceux couramment utilisés à Madagascar ou susceptibles d'y être employés. Certains produits utilisés depuis longtemps ont également été vérifiés dans un but comparatif.

\section{TRYPANBLEU}

Ce colorant a été le premier médicament actif sur un des hématozoaires en question, son activité sur $B$. bigemina ayant d'abord été signalée par NUTTALL et HADWEN (1909), activité confirmée par de nombreux auteurs. Il est actuellement remplacé dans la pratique par des piroplasmicides plus commodes à manipuler et ayant moins d'inconvénients; un résumé de nos résultats sera pourtant donné, le trypanbleu ayant une utilité au laboratoire dans certaines circonstances, quand il s'agit de guérir un accès à $B$. bigemina, sans influencer d'autres hématozoaires tel que $B$. argentina.

Un même lot de trypanbleu a été utilisé pour toutes les injections qui ont toujours été faites très lentement par la voie intraveineuse, avec une solution aqueuse à 1 p. 100 , préparée juste avant l'emploi.

\section{B. BIGEMINA}

\section{Pouvoir curatif}

Une seule injection de 1,0 à $7,1 \mathrm{mg} / \mathrm{kg}$ a toujours suffi à supprimer la première rechute après splénectomie d'un porteur (28 observations). Chez la majorité des animaux les parasites ne sont plus retrouvés sur frottis mince $24 \mathrm{~h}$. après le traitement, le sang de 26 sur 28 était négatif en moins de $48 \mathrm{~h}$; aucun des animaux n'a fait de rechute clinique par la suite, bien que les parasites réapparaissent après une période latente variable (de 2 à 
33 jours, en moyenne 14). Nous n'avons pas pu remarquer de corrélation entre la dose de trypanbleu et la rapidité de la réponse.

Par contre, seulement 11 animaux sur 20 (tous splénectomisés) ont été sauvés du premier accès après primo-infection par la seringue par une seule intervention (de 1,3 à $5,5 \mathrm{mg} / \mathrm{kg}$ ); les 9 autres sont morts au cours du premier accès (2) ou ont dû être retraités pendant cet accès (7); certains ont dû être retraités plus tard, lors de rechutes secondaires, et un d'entre eux mourut d'une telle rechute (non traitée) plus d'un mois après le premier traitement. Sur seulement 1 des 20 animaux le sang était négatif en moins de 24 h., sur seulement 7 en moins de $48 \mathrm{~h}$. Aucune corrélation apparente entre la dose de trypanbleu (dans les limites indiquées) et le résultat.

Dans la plupart des cas un seul traitement avec des doses comparables à celles indiquées plus haut amène rapidement la disparition des parasites lors de rechutes secondaires chez les splénectomisés, sans qu'il soit possible de savoir si cela est uniquement dû au traitement ou si la rechute aurait régressé spontanément. Dans d'autres cas la parasitémie ne diminue que très lentement.

\section{Pouvoir stérilisant}

Dans tous les cas où il a été possible de suivre les animaux quelque temps après le traitement, les parasites ont fait leur réapparition dans le sang, ou bien il a été possible de transmettre le parasite avec leur sang, et cela quelle que soit la dose de trypanbleu employée.

\section{B. ARGENTINA, A. MARGINALE et $A$. CENTRALE}

Nous n'avons pas fait d'expériences spéciales, mais des observations faites à plusieurs reprises, lorsqu'un accès à $B$. bigemina traité au trypanbleu coïncidait avec un accès à un des autres parasites mentionnés, confirment les conclusions de DONATIEN et LESTOQUARD 1927, SERGENT et al. (1945), et d'autres auteurs, à savoir que le trypanbleu est dépourvu d'activité $\operatorname{sur} B$. argentina et les anaplasmes.

\section{Toxicité}

Aux doses employées, le trypanbleu ne donne que rarement lieu à des accidents, s'il est administré en solution fraîche à 1 p. 100 et si l'injection est faite lentement.

Sur plus de 50 animaux traités au moins une fois, seulement 3 ont montré une nette intolérance: hypersalivation, respiration accélérée, tremblements et même prostration passagère. Les réactions se produisaient pendant ou quelques secondes après l'injection et sont directement imputables à la toxicité du trypanbleu. Les doses dans ces 3 cas variaient de 1,5 à $3,5 \mathrm{mg} / \mathrm{kg}$; dans un des cas l'animal commençait la réaction au cours de l'injection lente dès que la dose atteignait $1,5 \mathrm{mg} / \mathrm{kg}$, et cela 2 jours de suite.

\section{Conclusions sur le trypanbleu}

Le trypanbleu n'est plus indiqué dans la pratique: injection obligatoirement intraveineuse, résultats inconstants aux faibles doses (tout au moins chez les splénectomisés), tandis que des fortes doses causent d'après DONATIEN et LESTOQUARD (1927) des accidents fréquents et entraînent, du fait de la coloration de la viande, la saisie de la carcasse si l'animal doit être abattu d'urgence. De plus, le produit n'est actif que sur $B$. bigemina; un médicament polyvalent est préférable dans la pratique, le diagnostic spécifique de l'espèce de Babesia en cause ne pouvant alors pas être posé sans microscope.

Ce colorant reste cependant utilisable au laboratoire lors d'une prospection des hématozoaires par splénectomie, quand il est nécessaire de guérir l'animal de sa rechute à $B$. bigemina sans pour autant influencer d'autres parasites sanguins. Il est très efficace pour supprimer cette première rechute, même à des doses aussi faibles que 1 à $2 \mathrm{mg} / \mathrm{kg}$, sans que lanimal soit stérilisé de son infection.

Les résultats sont beaucoup moins réguliers s il s’agit de guérir des animaux splénectomisés après primo-infection, si l'on veut vérifier qu'une souche de $B$. bigemina $n$ 'est pas contaminée par $B$. argentina par exemple; dans ces circonstances la Pentamidine nous semble préférable (voir plus loin).

Notons que la résistance au trypanbleu signalée par RAYNAUD (1962) nous semble pouvoir être expliquée par le fait que la première rechute après splénectomie d'un porteur répond toujours très bien au trypanbleu à l'opposé du premier accès après primo- 
infection. Nous n'avons rencontré aucune souche que l'on pourrait appeler résistante au trypanbleu de façon constante; il a été observé à plusieurs reprises qu'une même souche qui répondait très bien au trypanbleu chez un animal donné, n'y répondait pas chez un autre.

\section{EUFLAVINE (= Gonacrine, N.D.)}

Il semble que STEPHAN et ESQUIBEL (1929) aient été les premiers à rapporter l'efficacité de ce produit contre la piroplasmose bovine; leurs résultats ont été confirmés par de nombreux auteurs. D'autres produits plus faciles à administrer l'ont actuellement remplacé en grande partie, mais il conserve une bonne réputation pour le traitement de la babésiellose ( $B$. argentina); aussi nous ne donnerons les détails que sur les essais de traitement de ce parasite.

Toutes les expériences ont été faites avec la solution commerciale de Gonacrine à 5 p. 100 , administrée par injection lente en intraveineuse.

\section{B. BIGEMINA}

\section{Pouvoir curatif}

Cinq bovins splénectomisés ont été traités Iors du premier accès après primo-infection par la seringue. Des doses de 0,8 et $2,1 \mathrm{mg} / \mathrm{kg}$ se sont avérées être insuffisantes; il n'y avait dans les deux cas aucune influence sur la parasitémie, l'aspect des parasites, la température et l'état clinique pendant les $24 \mathrm{~h}$. suivant le traitement; l'animal traité à $0,8 \mathrm{mg} / \mathrm{kg}$ est mort de l'accès le lendemain du traitement, l'autre a été retraité avec un autre piroplasmicide. Les trois autres sujets ont reçu des doses de 2,9 - 3,1 et $5,4 \mathrm{mg} / \mathrm{kg}$ et ont été guéris de l'accès par cette seule intervention; le sang était négatif $48 \mathrm{~h}$. après le traitement, tandis qu'après $24 \mathrm{~h}$. de rares parasites d'aspect dégénéré persistaient encore $(*)$.

Les Babesiae sont réapparues dans les trois cas après des périodes latentes de 4 à 8 jours, et dans deux cas un autre traitement a été nécessaire étant donné l'importance de la rechute parasitaire et thermique.

\section{Pouvoir stérilisant}

Aucun dans tous les cas.

\section{B. ARGENTINA}

a) Animaux splénectomisés indemnes, traités lors du premier accès après primo-infection par la seringue,

(") Des frottis faits dans un cas 5 h. après le traitement ne révèlent plus que des parasites ayant un aspect dégénéré (chromatine agglomérée en boule), en nombre déjà bien diminué.

\begin{tabular}{|c|c|c|c|c|c|}
\hline & $\begin{array}{l}\text { Jours après } \\
\text { le traitement }\end{array}$ & Dose & Parasitémie & Température & Remarques \\
\hline Cas A & $\begin{array}{l}0 \\
1\end{array}$ & $4,4 \mathrm{mg} / \mathrm{kg}$ & $\begin{array}{l}+ \\
+\end{array}$ & $\begin{array}{l}3906 \\
\text { normale }\end{array}$ & $\begin{array}{l}\text { 4e jour de l'accès parasitaire, 3e de l'ac- } \\
\text { cès thermique. } \\
\text { Reçoit un autre piroplasmicide. }\end{array}$ \\
\hline Cas B & $\begin{array}{l}0 \\
1 \\
2\end{array}$ & $5,4 \mathrm{mg} / \mathrm{kg}$ & $\begin{array}{c}(+) \\
\text { très rares } \\
(+) \\
\text { très rares } \\
0\end{array}$ & $\begin{array}{c}3908 \\
40 \cdots 0 \\
\text { normale }\end{array}$ & $\begin{array}{l}20 \text { jour de l'accès parasitaire, 4e de l'ac- } \\
\text { cès thermique. }\end{array}$ \\
\hline Cas $\mathrm{C}$ & 0 & $6,7 \mathrm{mg} / \mathrm{kg}$ & $\begin{array}{c}(+) \\
\operatorname{assez} \text { rares } \\
0\end{array}$ & $\begin{array}{l}3909 \\
3708\end{array}$ & $\begin{array}{l}\text { 4e jour de l'accès parasitaire et ther- } \\
\text { mique (*) } \\
\text { Mort avec des symptômes nerveux, hu- } \\
\text { bésiellose cérébrale (*). }\end{array}$ \\
\hline Cas D & 0 & $10,0 \mathrm{mg} / \mathrm{kg}$ & $\begin{array}{c}+ \\
(+) \\
\text { très rares }\end{array}$ & $\begin{array}{l}4001 \\
3908\end{array}$ & $\begin{array}{l}4^{\mathrm{e}} \text { jour de l'accès parasitaire et ther- } \\
\text { mique. } \\
\text { Reçoit un autre piroplasmicide. }\end{array}$ \\
\hline
\end{tabular}

(*) L'animal a été traité 2 jours plus tôt sans résultat à la Pentamidine.
(**) Capillaires du cortex cérébral emplis d’érythrocytes infestés; aucun parasite n'est trouvé dans les calques d'autres organes et dans le sang. La mort survient moins de $24 \mathrm{~h}$. après le traitement. 
b) Animaux normaux traités lors du premier accès après primo-infection par la seringue.

\begin{tabular}{|c|c|c|c|c|c|}
\hline & $\begin{array}{l}\text { Jours après } \\
\text { le traitement }\end{array}$ & Dose & Parasitémie & Température & Remarques \\
\hline Cas $A$ & 0 & $1,9 \mathrm{mg} / \mathrm{kg}$ & $\begin{array}{l}(+) \\
\text { 1rès rares } \\
(+) \\
\text { très rares }\end{array}$ & $\begin{array}{l}3908 \\
3903\end{array}$ & $\begin{array}{l}\text { Frison adulte importé. } \\
\text { ter jour de l'accès parasitaire et ther- } \\
\text { mique. } \\
\text { Reçoit au autre piroplasmicide. }\end{array}$ \\
\hline Cas B & $\begin{array}{c}0 \\
1 \\
2-3\end{array}$ & $\begin{array}{l}2,2 \mathrm{mg} / \mathrm{kg} \\
2,2 \mathrm{mg} / \mathrm{kg}\end{array}$ & $\begin{array}{c}(+) \\
\text { assez rares } \\
(+) \\
\text { très rares } \\
(+) \\
\text { très rares }\end{array}$ & $\begin{array}{l}4004 \\
40 \times 0 \\
\text { normale }\end{array}$ & $\begin{array}{l}\text { Frison aldulte importé. } \\
\text { jer jour de l'accès parasıtaire, } 3 \text { e de l'ac- } \\
\text { cès thermique. } \\
\text { Reçoit un autre piroplasmicide au jour } 3 \text {. }\end{array}$ \\
\hline Cas C & $\begin{array}{l}0 \\
1 \\
2\end{array}$ & $2,3 \mathrm{mg} / \mathrm{kg}$ & $\begin{array}{l}(+) \\
\text { assez rares } \\
(+) \\
\text { très rares } \\
0\end{array}$ & $\begin{array}{l}4100 \\
\text { normale }\end{array}$ & $\begin{array}{l}\text { Frison adulte importé. } \\
1 \text { er jour de l'accès parasitaire, } 4^{\circ} \text { de l'ac- } \\
\text { cès thermique. }\end{array}$ \\
\hline Cas D & $\begin{array}{l}0 \\
1 \\
2\end{array}$ & $3,3 \mathrm{mg} / \mathrm{kg}$ & $\begin{array}{l}(+) \\
\text { assez rares } \\
0 \\
0\end{array}$ & $\begin{array}{l}4000(*) \\
3901(\cdot) \\
\text { normale }\end{array}$ & $\begin{array}{l}\text { Veau normánd. } \\
\text { 2e jour de l'accès parasitaire ( }) \text {. }\end{array}$ \\
\hline
\end{tabular}

(*) L'accès thermique est faussé par un accès concomittant d'Eperythrozoon reganudes Hoyte, 1962, qui persistent encore le lendemain du traitement

c) Animaux splénectomisés, traités lors d'une rechute secondaire importante.

Quatre observations. Dans tous les cas l'injection de 4,6 à $8,0 \mathrm{mg} / \mathrm{kg}$ a ramené la température à une valeur normale ou presque normale en moins de $24 \mathrm{~h}$.; les parasites avaient disparu en moins de $24 \mathrm{~h}$. dans un cas, en moins de $48 \mathrm{~h}$. dans tous les cas.

\section{Pouvoir stérilisant}

Apparemment aucun; dans six cas au mons les parasites ont fait leur réapparition après des doses de 2,2 à $8,0 \mathrm{mg} / \mathrm{kg}$, aussi bien sur des animaux normaux que sur des splénectomisés. Un autre exemple est fourni par un bovin splénectomisé, subissant un accès après primoinfection par des tiques. Voici le tableau des traitements effectués à partir du premier jour de l'accès parasitaire, tableau qui illustre, outre le manque de pouvoir stérilisant de la Gonacrine, en même temps le peu de sensibilité à différents piroplasmicides de $B$. argentina transmise par les tiques:

\section{TABLEAU}

\begin{tabular}{|c|c|c|c|}
\hline $\begin{array}{c}\text { Jour } \\
\text { de l'accès } \\
\text { thermique }\end{array}$ & $\begin{array}{l}\text { Tempé- } \\
\text { rature }\end{array}$ & $\begin{array}{l}\text { Para- } \\
\text { sitémie }\end{array}$ & $\begin{array}{l}\text { Traitement } \\
(\mathrm{mg} / \mathrm{kg})\end{array}$ \\
\hline 2 & $40^{0} 6$ & $(+)$ & Lomidıne: 2,7 \\
\hline 3 & $40 " 8$ & + & $\begin{array}{c}\text { Zothélone : } 0,7 \\
\text { et Gonacrine : } 3,3\end{array}$ \\
\hline 4 & 3907 & + & $\begin{array}{c}\text { Zothélone : } 0,7 \\
\text { et Gonacrine : } 3,3\end{array}$ \\
\hline 5 & 3806 & non & - \\
\hline 6 & $39 \circ 0$ & $\underset{(+)}{e x a m i n e}$ & Gonacrine: 3,3 \\
\hline 7 à 12 & normale & 0 & - \\
\hline 13 & 3901 & $(+)$ & Zothélone : 0,7 \\
\hline 14 & $38^{\circ} 7$ & $(+)$ & $\begin{array}{l}\text { Zothélone : } 1,0 \\
\text { (en } 2 \text { fractions) }\end{array}$ \\
\hline 15 & 3900 & $(+)$ & Gonacrine: 3,3 \\
\hline 16 & 3800 & 0 & Gonacrine : 5,0 \\
\hline 17 & $39 \times 6$ & 0 & Gonacrine : 5,0 \\
\hline 18 & $38^{\circ} 0$ & 0 & Gonacrine : 5,0 \\
\hline
\end{tabular}


L'animal meurt le $18^{2}$ jour avec présence de rares $B$. argentina dans les érythrocytes des capillaires du cortex cérébral ! (Il est probablement mort d'intoxication par la Gonacrine après avoir présenté maigreur, inappétence et abattement au cours des deux jours précédant sa mort.)

\section{A. MARGINALE ET A. CENTRALE}

Aucune expérience spéciale, mais des observations faites par coïncidence n'ont permis de constater aucune action de la Gonacrine sur les anaplasmes, ce qui confirme les résultats obtenus par Rampon (1933).

\section{Toxicité}

Aux doses employées et administrée une seule ou deux fois, la Gonacrine n’a pas provoqué de symptômes d'intoxication. Une exception doit être faite, peut-être, pour l'animal traité à $10 \mathrm{mg} / \mathrm{kg}$ (voir sous le traitement de $B$. argentina); il a maigri après l'intervention, a perdu son appétit, ne pouvait plus se lever à partir du $11^{\circ}$ jour, et a dû être sacrifié à l'agonie 13 jours après l'injection; la cause de la maladie n'a pas été élucidée, aucun parasite n'a été trouvé sur les calques et frottis des divers organes (y compris le cortex cérébral); le cadavre était étique, anémique et le contenu du rectum diarrhéique. (Voir aussi PANISSET et GRASSET (1931) qui rapportent la toxicité à retardement de la Gonacrine pour les lapins, morts avec de la diarrhée.)

Des doses élevées et répétées sont vraisemblablement dangereuses (voir le cas relatêt ci-dessus sous le pouvoir stérilisant sur B. argentina.)

\section{Conclusions sur l'euflavine}

Bonne action piroplasmicide sur $B$. bigemina à $3 \mathrm{mg} / \mathrm{kg}$ environ, tandis que les doses de 1 à $2 \mathrm{mg} / \mathrm{kg}$ ne semblent pas suffire (tout au moins sur les splénectomisés).

La Gonacrine a une bonne réputation dans la pratique pour le traitement de la babésiellose (B. argentina); il existe certainement une action sur ce parasite, mais elle a été quelque peu décevante et lente. (A noter que le produit donnerait également de bons résultats dans la pratique sur la nuttalliose équine, tandis qu'il semblait dépourvu d'activité directe sur le parasite chez un animal splénectomisé (UILENBERG, 1967)).
La Gonacrine ne stérilise pas les animaux des infections à Babesia.

Elle ne possède pas d'activité sur les anaplasmes.

Le produit semble sans danger aux doses normales, mais il a le grand inconvénient de devoir être injecté obligatoirement par voie intraveineuse (surtout difficile en élevage extensif, avec des animaux demi-sauvages) et il colore également temporairement les tissus de lanimal en jaune, ce qui peut amener des saisies de carcasses si l'animal doit être abattu d'urgence. Etant donné ces inconvénients et le fait que sa réputation dans le traitement de la babésiellose semble quelque peu exagérée, d'autres piroplasmicides plus faciles à manipuler et ayant une activité au moins comparable (voir plus loin) nous semblent actuellement préférables dans la pratique.

\section{SULFATE DE QUINURONIUM (Acaprine, N.D., Zothélone, N.D., etc.)}

L'activité de ce produit contre les babésioses bovines a été reconnue à partir de 1935 (CERNAIANU et al., 1935 a $1935^{\mathrm{b}}$, CERRUTI et FANTONI, 1935, Anonyme, 1935 et de nombreux auteurs par la suite). L'activité sur $B$. bigemina étant suffisamment connue, nous ne donnerons pas les détails de nos expériences; les résultats irréguliers obtenus sur $B$. argentina seront donnés en détail.

Nos expériences ont été faites avec la solution commerciale à 5 p. 100 , soit le Zothélone (qui n'est actuellement plus fabriqué), soit l'Acaprine; les injections ont toujours été faites par voie sous-cutanée.

\section{B. BIGEMINA}

\section{Pouvoir curatif}

Sept bovins splénectomisés ont été traités lors du premier accès après primo-infection, par la seringue dans six cas, par les tiques dans un cas. Les doses ont varié de 0,3 à $0,6 \mathrm{mg} / \mathrm{kg}$. Ces doses relativement faibles ont un effet rapide sur les parasites, qui ont un aspect dégénéré le lendemain du traitement $\left({ }^{*}\right)$; le sang est négatif en moins de $48 \mathrm{~h}$, sauf dans un

(*) Un contrôle effectué 4 heures après un traitement à $0,4 \mathrm{mg} / \mathrm{kg}$ a montré que la plupart des parasites sont déjà dégénérés à ce moment. 
cas traité à $0,4 \mathrm{mg} / \mathrm{kg}$ où cela a pris 3 jours, mais jamais en moins de $24 \mathrm{~h}$. L'effet sur l'hyperthermie est lente aux doses inférieures à $0,5 \mathrm{mg} / \mathrm{kg}$, où elle a mis 2 jours pour disparaître; la température est devenue normale le lendemain des traitements à 0,5 et $0,6 \mathrm{mg} / \mathrm{kg}$. Des rechutes importantes, nécessitant une deuxième intervention 10 et 12 jours après la première, se sont produites dans deux cas.

Six animaux splénectomisés ont été traités lors de rechutes secondaires; on a pu constater l'effet direct d'une dose de $0,25 \mathrm{mg} / \mathrm{kg}$ (deux cas) par l'aspect dégénéré que présentaient les parasites le lendemain. Un sujet, traité à $0,7 \mathrm{mg} / \mathrm{kg}$, a fait une rechute mortelle. Dans les cas traités à $0,6-0,8 \mathrm{mg} / \mathrm{kg}$ le sang était négatif en moins de 24 h., tandis que les parasites n'avaient disparu qu'après 2 jours après des doses de 0,25 et $0,5 \mathrm{mg} / \mathrm{kg}$.

Nous n'avons pu constater aucune différence entre l'activité de l'Acaprine et du Zothélone.

\section{Pouvoir stérilisant}

Aucun aux doses employées, dans 11 cas sur 13 au moins les parasites ont fait leur réapparition, même après $0,8 \mathrm{mg} / \mathrm{kg}$.
Une expérience a été faite sur un animal non splénectomisé, porteur de $B$. bigemina; les parasites ont fait leur réapparition dans le sang 3 semaines après l'administration de Zothélone à $2,1 \mathrm{mg} / \mathrm{kg}$ (et ils ont également fait une sortie après la splénectomie 3 mois et demi plus tard). Dans un autre cas, un bovin splénectomisé traité à $1,0 \mathrm{mg} / \mathrm{kg}$ pour un accès à $B$. argentina (primo-infection par tiques) a néanmoins fait un accès à $B$. bigemina, transmise en même temps que $B$. argentina; l'accès a simplement été retardé.

Il ne semble donc pas qu'une stérilisation puisse être obtenue aux doses normalement employées $(0,5$ à $1,0 \mathrm{mg} / \mathrm{kg})$. Par contre, LEGG (1936) trouve que les animaux sont stérilisés de leur infection après traitement à l'Akiron (= Acaprine), (administré par la voie intraveineuse il est vrai).

\section{B. ARGENTINA}

\section{Pouvoir curatif}

a) Animaux splénectomisés, traités lors du premier accès après primo-infection par la seringue.

\begin{tabular}{|c|c|c|c|c|c|}
\hline & $\begin{array}{l}\text { Jours après } \\
\text { le traitement }\end{array}$ & Dose & Parasitémie & Température & Remarques \\
\hline Cas $\mathrm{A}$ & $\begin{array}{l}0 \\
1 \\
2 \\
3 \\
4 \\
5 \\
6\end{array}$ & $\begin{array}{c}0,25 \mathrm{mg} / \mathrm{kg} \\
\text { (Acaprine) } \\
\begin{array}{c}0,7 \mathrm{mg} / \mathrm{kg} \\
(\text { Ac. })\end{array} \\
1,0 \mathrm{mg} / \mathrm{kg} \\
(\text { Ac. })\end{array}$ & $\begin{array}{l}0 \\
0 \\
(+) \\
\text { très rares } \\
(+) \\
\text { très rares } \\
(+) \\
(+) \\
0\end{array}$ & $\begin{array}{c}\text { normale } \\
» \\
39 \circ 3 \\
39 \circ 8 \\
40 \circ 0 \\
\text { normale }\end{array}$ & $\begin{array}{l}\text { Administré pendant l'ìncubation. } \\
\begin{array}{l}\text { Dose totale, répartie en } 2 \text { prises à } 2 \mathrm{~h} \text {. } \\
\text { d'intervalle. }\end{array}\end{array}$ \\
\hline Cas B & $\begin{array}{l}0 \\
1\end{array}$ & $\begin{array}{l}0,6 \mathrm{mg} / \mathrm{kg} \\
\text { (Ac.) }\end{array}$ & $\begin{array}{c}(+) \\
\text { très rares } \\
0\end{array}$ & $\begin{array}{c}4000 \\
\text { normale }\end{array}$ & 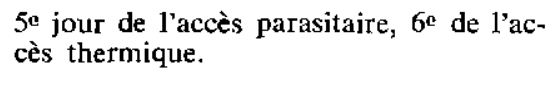 \\
\hline Cas C & $\begin{array}{l}0 \\
1\end{array}$ & $\begin{array}{l}1,0 \mathrm{mg} / \mathrm{kg} \\
\text { (Zothélone) }\end{array}$ & $\begin{array}{c}(+) \\
\text { très rares } \\
0\end{array}$ & $\begin{array}{c}40 \times 0 \\
\text { normale }\end{array}$ & $\begin{array}{l}\text { Jer jour de l'accès parasitaire et ther- } \\
\text { mique. }\end{array}$ \\
\hline
\end{tabular}


b) Animaux splénectomisés indemnes, traités

lors du premier accès après primo-infection par les tiques.

\begin{tabular}{|c|c|c|c|c|c|}
\hline & $\begin{array}{c}\text { Jours après } \\
\text { le traitement }\end{array}$ & Dose & Parasitémie & Température & Rernarques \\
\hline Cas A & $\begin{array}{l}0 \\
1 \\
2\end{array}$ & $\begin{array}{l}0,5 \mathrm{mg} / \mathrm{kg} \\
(\text { Zoth.) } \\
0,75 \mathrm{mg} / \mathrm{kg} \\
\text { (Zoth.) }\end{array}$ & $\begin{array}{l}(+) \\
\text { rares } \\
(+) \\
\text { rares } \\
(+) \\
\text { rares }\end{array}$ & $\begin{array}{l}40 \circ 7 \\
40 \circ 0 \\
40 \circ 3\end{array}$ & $\begin{array}{l}\text { 2e jour de l'accès parasitaire, 3" de l'ac- } \\
\text { cès thermique. } \\
\text { Reçoit un autre piroplasmicide. }\end{array}$ \\
\hline Cas B & $\begin{array}{l}0 \\
1 \\
2\end{array}$ & $\begin{array}{l}0,5 \mathrm{mg} / \mathrm{kg} \\
\text { (Zoth.) } \\
0,7 \mathrm{mg} / \mathrm{kg} \\
\text { (Zoth.) }\end{array}$ & $\begin{array}{l}(+) \\
\text { rares } \\
+ \\
+\end{array}$ & $\begin{array}{l}40 \div 6 \\
4008 \\
3907\end{array}$ & 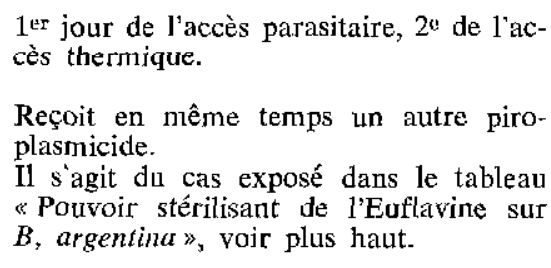 \\
\hline Cas C & $\begin{array}{c}0 \\
1 \\
2-5 \\
6\end{array}$ & $\begin{array}{l}1,0 \mathrm{mg} / \mathrm{kg} \\
\text { (Zoth.) }\end{array}$ & $\begin{array}{l}(+) \\
\text { très ráres } \\
(+) \\
\text { très rares } \\
(+) \\
\text { très rares } \\
\quad(+) \\
\text { pas rares }\end{array}$ & $\begin{array}{c}\text { normale } \\
3903 \\
\text { oscille autour } \\
\text { de } 400 \\
4002\end{array}$ & $\begin{array}{l}\text { 1er jour de l'accès parasitaire. } \\
\text { Reçoit un autre piroplasmicide. }\end{array}$ \\
\hline Cas D & $\begin{array}{l}0 \\
1 \\
2\end{array}$ & $\begin{array}{l}1,0 \mathrm{mg} / \mathrm{kg} \\
\text { (Zoth.) }\end{array}$ & $\begin{array}{l}+ \\
(+) \\
\text { très rares } \\
0\end{array}$ & $\begin{array}{c}40^{\circ 0} \\
3903 \\
\text { normale }\end{array}$ & $\begin{array}{l}\text { 1er jour de l'accès clinique (inappétence, } \\
\text { abattement), le sang n'a pas été examıné } \\
\text { plus tôt. Dose administrêe en } 2 \text { fractions. }\end{array}$ \\
\hline Cas $\mathrm{E}$ & $\begin{array}{l}0 \\
1 \\
2 \\
3\end{array}$ & $\begin{array}{l}1,0 \mathrm{mg} / \mathrm{kg} \\
(\mathrm{Zoth} .) \\
0,7 \mathrm{mg} / \mathrm{kg} \\
\text { (Zoth.) }\end{array}$ & $\begin{array}{c}+ \\
++ \\
0 \\
0\end{array}$ & $\begin{array}{c}4103 \\
40^{\circ} 5 \\
39{ }^{\prime} 3 \\
\text { normale }\end{array}$ & $\begin{array}{l}\text { 1er jour de l'accès clinique (comme } \\
\text { cas D). Dose administrée en } 2 \text { fractions. }\end{array}$ \\
\hline
\end{tabular}

c) Animal splénectomisé, traité lors d'une rechute secondaire importante.

Un cas : $0,7 \mathrm{mg} / \mathrm{kg}$ (Acaprine) au premier jour de la rechute parasitaire, $2^{\mathrm{e}}$ jour de l'hyperthermie. Parasitémie très faible, température $39,8^{\circ}$. L'animal est trouvé mort le lendemain, avec d'assez nombreuses $B$. argentina dans les capillaires du cortex cérébral. (Le sujet était dans un état cachectique.)

d) Animaux normaux, traités lors d'un accès par primo-infection transmise par la seringue.

\begin{tabular}{|c|c|c|c|c|c|}
\hline & $\begin{array}{c}\text { Jours après } \\
\text { le trattement }\end{array}$ & Dose & Parasitémie & Température & Remarques \\
\hline Cas A & 0 & $\begin{array}{c}0,7 \mathrm{mg} / \mathrm{kg} \\
\text { (Zoth. })\end{array}$ & + & 4000 & $\begin{array}{l}\text { Frison adulte importé. } \\
\text { 2e jour de l'accès parasitaire, 10r jour } \\
\text { de 1'accès thermique, }\end{array}$ \\
\hline Cas B & 0 & $\begin{array}{c}1,0 \mathrm{mg} / \mathrm{kg} \\
(\text { Zoth. })\end{array}$ & $\begin{array}{c}(+) \\
\text { très rares } \\
\text { normale }\end{array}$ & $\begin{array}{c}40^{\circ} 1 \\
\text { normale }\end{array}$ & $\begin{array}{l}\text { Veau normand. } \\
\text { 2e jour de l'accès parasitaire, 4e jour de } \\
\text { l'accès thermique. }\end{array}$ \\
\hline
\end{tabular}




\section{Pouvoir stérilisant}

Probablement aucun, même après $1 \mathrm{mg} / \mathrm{kg}$. (Voir aussi le tableau sous «Pouvoir stérilisant» de l'euflavine sur $B$. argentina, plus haut.)

\section{A. MARGINALE ET A. CENTRALE}

Aucune expérience spéciale, mais des observations faites par coïncidence confirment l'opinion de nombreux auteurs, à savoir que le produit n’a aucune action sur les anaplasmes.

\section{Toxicité}

Normalement le produit est sans grand danger aux doses normales, bien que des symptômes d'intoxication soient fréquents, même après des doses relativement faibles. Ainsi nous avons traité plus de 500 bovins normaux au Zothélone à $0,5 \mathrm{mg} / \mathrm{kg}$ (comme traitement systématique après la prémunition artificielle à $B$. bigemina); les réactions ont commencé environ 15 minutes après l'injection. Plus de la moitié des animaux salivaient, sans autre symptôme. Dans 5 p. 100 des cas environ les animaux tremblaient, se couchaient et présentaient un écoulement séreux des narines et de très rares animaux avaient en outre les membres raides, expulsaient fréquemment leurs excréments et se montraient inquiets. Ces symptômes étaient passagers chez tous les animaux et avaient disparu une heure après environ.

Ajoutons que la dose de $2,75 \mathrm{mg} / \mathrm{kg}$ est souvent mortelle (CALLOW et MELLORS, 1966; CALLOW et Mc GREGOR, 1967) et que même aux doses normales de 0,5 à $0,8 \mathrm{mg} / \mathrm{kg}$ employées par CALLOW et MELLORS des mortalités sont signalées. La marge de sécurité entre la dose efficace sur les Babesiae et la dose mortelle est faible.

\section{Conclusions sur le sulfate de quinuronium}

Apparemment aucune différence d'activité entre les deux présentations employées (Acaprine et Zothélone). Produit très actif sur $B$. bigemina, même des doses aussi faibles que 0,25 à $0,5 \mathrm{mg} / \mathrm{kg}$ sont actives. L'activité sur $\boldsymbol{B}$. argentina nous a en fait déçu; elle exıste, mais est irrégulière, et la dose requise est plus élevée que celle pour $B$. bigemina. (Rappelons que TCHERNOMORETZ (1943) ne voit aucun résultat avec une dose de $2,8 \mathrm{mg} / \mathrm{kg}$
d'Acaprine sur $B$. berbera ( $=B$. argentina). Aucune activité sur les anaplasmes. Les animaux ne sont pas stérilisés de leurs Babesiae.

Les avantages de ce produit sont sa facilité d'emploi (voie sous-cutanée) et son prix peu élevé; il possède par ailleurs une certaine toxicité et il convient donc de l'utiliser avec prudence.

\section{PENTAMIDINE (= Lomidine, N.D.)}

Ce produit a été essayé d'abord par LOURIE et YORKE (1939) contre la piroplasmose canine, et par GILBERT et AVELANGE (1947) contre la piroplasmose équine. TCHERNOMORETZ (1943) rapporte l'inefficacité de la Pentamidine contre $B$. berbera $(=B$. argentina). Le fabricant préconise son emploi contre toutes les babésioses des bovins, sans que nous ayons pu trouver de référence précise quant aux résultats.

BUCK et al. (1951) trouvent qu'elle donne de bons résultats, associée à la Gonacrine, contre l'anaplasmose bovine, mais RAYNAUD (1962) ne peut pas le confirmer.

Les résultats obtenus étant nets, nous n’en donnerons qu un résumé, avec quelques exemples en détail.

Les expériences ont toujours été faites avec la solution commerciale à 4 p. 100 de Lomidine, administrée par voie intramusculaire, sauf indication contraire.

\section{B. BIGEMINA}

\section{Pouvoir curatif}

a) Animaux splénectomisés, traités lors du premier accès après primo-infection par la seringue, dans un cas par les tiques.

La dose de $0,8 \mathrm{mg} / \mathrm{kg}$ (administrée par voie intraveineuse) s'est montrée insuffisante, la parasitémie n’ayant presque pas diminué après $24 \mathrm{~h}$. Sept animaux ont été traités (par voie intramusculaire) avec des doses de 1,1 à $5,3 \mathrm{mg} / \mathrm{kg}$; le résultat a été rapide dans tous les cas, y compris un animal infecté par les tiques et traité à $1,6 \mathrm{mg} / \mathrm{kg}$. Le sang était négatif en moins de $24 \mathrm{~h}$. dans un cas $(5,3 \mathrm{mg} / \mathrm{kg})$, en moins de $48 \mathrm{~h}$. dans quatre 
cas $(1,3$ à $3,4 \mathrm{mg} / \mathrm{kg})$ et en moins de 3 jours dans deux cas $(1,1$ et $2,4 \mathrm{mg} / \mathrm{kg})$. Dans tous les cas où le sang n'était pas encore négatif après 24 h., les parasites étaient devenus rares ou très rares, et souvent d'un aspect dégénéré. Lors- qu'elle existait au moment du traitement, l'hyperthermie avait disparu ou presque en moins de $24 \mathrm{~h}$.

Voici un exemple d'un animal in extremis sauvé par la Lomidine:

\begin{tabular}{|c|c|c|c|c|}
\hline $\begin{array}{l}\text { Jour après } \\
\text { le traitement }\end{array}$ & Dose & Parasitémie & Température & Remarques \\
\hline $\begin{array}{l}0 \\
1 \\
2 \\
3\end{array}$ & $2,4 \mathrm{mg} / \mathrm{kg}$ & $\begin{array}{c}+++ \\
(+) \\
\text { très rares } \\
(+) \\
\text { très rares } \\
0\end{array}$ & $\begin{array}{c}\underset{3705}{\text { normale }}\left({ }^{*}\right) \\
\text { normale } \\
»\end{array}$ & $\begin{array}{l}\text { Hémoglobinurie, abattement }\left({ }^{*}\right) \text {. } \\
\text { Encore très abattu. } \\
\text { Appétit revenu. } \\
\text { Guérison. }\end{array}$ \\
\hline
\end{tabular}

(*) L'animal a présenté une forte hyperthermie pendant les 2 jours précédents (de $40 \circ 0$ et 4103 ), avec hémoglobinurie; il était dans la phase terminale d'un accès très grave.

b) Animaux splénectomisés, traités lors d'une rechute secondaire importante.

Huit animaux traités avec des doses de 1,0 à $4,0 \mathrm{mg} / \mathrm{kg}$. Dans tous les cas le sang était négatif le lendemain ou le surlendemain du traitement, et dans les deux cas où la rechute fut accompagnée d'une hyperthermie, la température était normale le lendemain du traitement (à 1,0 et $4,0 \mathrm{mg} / \mathrm{kg}$ ).

c) Animaux normaux, traités lors du premier accès après infection par la seringue.

Neuf animaux ayant des parasitémies variables de peu importantes à très importantes, presque tous ayant des températures situées entre $40^{\circ}$ et $41^{\circ}$. Traitement à $2,4 \mathrm{mg} / \mathrm{kg}$ (un cas), $3,0 \mathrm{mg} / \mathrm{kg}$ (six cas), $4,0 \mathrm{mg} / \mathrm{kg}$ (un cas) et $6,0 \mathrm{mg} / \mathrm{kg}$ (un cas). Dans tous les cas le sang était négatif en moins de $24 \mathrm{~h}$. et la température était normale dans le même temps (sauf dans un cas où elle était faussée par une sortie d'Eperythrozoon teganodes).

\section{Pouvoir stérilisant}

Probablement aucun aux doses employées. En effet, dans onze cas de traitement de splénectomisés nous avons pu vérifier que l'animal n'était pas stérilisé de l'infection après des doses de 1,0 à $4,0 \mathrm{mg} / \mathrm{kg}$. De même pour trois animaux normaux, traités à $2,4-4,0$ et $4,0 \mathrm{mg} / \mathrm{kg}$ (la persistance de l'état de prémunition a été vérifiée dans un cas par splénec- tomie deux semaines après le traitement, dans deux cas par la réapparition des parasites dans le sang).

\section{B. ARGENTINA}

\section{Pouvoir curatif}

a) Animaux splénectomisés, traités lors de la primo-infection transmise par la seringue.

Deux cas, traités à 4,0 et $6,5 \mathrm{mg} / \mathrm{kg}$. Aucune influence sur la parasitémie ni sur l'hyperthermie, pendant $48 \mathrm{~h}$. Un animal est mort trois jours après ce traitement avec des symptômes nerveux, et les capillaires du cortex cérébral étaient emplis d’érythrocytes infestés. L’autre est donné comme exemple $A$ plus loin.

b) Animaux splénectomisés, traités lors d'une primo-infection par les tiques.

Deux cas, traités à 1,6 et $2,7 \mathrm{mg} / \mathrm{kg}$. Dans le premier cas la température, normale le jour du traitement, s'est élevée à partir du lendemain, pour se maintenir autour de $40^{\circ}$ jusqu'au $6^{\circ}$ jour, quand l'animal a reçu un autre piroplasmicide; la parasitémie est restée inchangée pendant 5 jours après le premier traitement, mais a augmenté le $6^{\mathrm{e}}$ jour. Le deuxième cas est donné en exemple plus loin; là encore la Lomidine n'avait apparemment aucune influence sur l'évolution de la maladie.

c) Animaux normaux, traités lors du premier accès après infection par la seringue. 
Des doses de $2,7 \mathrm{mg} / \mathrm{kg}$ n'ont eu aucune influence apparente sur l'évolution chez trois veaux frisons; un autre piroplasmicide a dû être administré 2 jours plus tard.

Dans un seul cas la Lomidine a semblé agir : Zébu. $5,8 \mathrm{mg} / \mathrm{kg}$ au premier jour de l'accès thermique (le début de l'accès parasitaire étant inconnu). Parasitémie très faible, température $40,0^{\circ}$. Le sang est négatif le lendemain et la température est normale. On ne sait pas si l'animal était indemne ou non de $B$. argentina avant l'inoculation de notre souche.

\section{Exemples:}

Exemple A

Primo-infection transmise par la seringue. Animal splénectomisé

\begin{tabular}{|c|c|c|c|c|}
\hline $\begin{array}{l}\text { Jours après } \\
\text { le traitement }\end{array}$ & Dose & Parasitémie & Température & Remarques \\
\hline $\begin{array}{l}0 \\
1 \\
2\end{array}$ & $6,5 \mathrm{mg} / \mathrm{kg}$ & $\begin{array}{l}++ \\
++ \\
++\end{array}$ & $\begin{array}{l}40 \% 9 \\
40 \circ 4 \\
40 \% 4\end{array}$ & 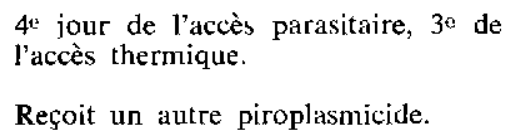 \\
\hline
\end{tabular}

Exemple B

Primo-infection transmise par les tiques. Animal splénectomisé

\begin{tabular}{|c|c|c|c|c|}
\hline $\begin{array}{c}\text { Jours après } \\
\text { le traitement }\end{array}$ & Dose & Parasitémie & Température & Remarques \\
\hline 0 & $2,7 \mathrm{mg} / \mathrm{kg}$ & $\begin{array}{c}(+) \\
\text { tares } \\
+\end{array}$ & 4006 & $\begin{array}{c}\text { Premier jour de l'accès parasıtaire, } \\
\text { 2e de l'accès thermique. } \\
\text { Reçoit d'autres piroplasmicides. }\end{array}$ \\
\hline
\end{tabular}

\section{Pouvoir stérilisant}

Aucun, même à la dose de $6,5 \mathrm{mg} / \mathrm{kg}$.

\section{A. MARGINALE ET A, CENTRALE}

Aucune expérience n'a été faite spécialement, mais quelques observations faites par coïncidences confirment les observations de RAYNAUD (1962) qui ne voit aucun effet de la Lomidine sur ces deux parasites.

\section{Toxicité}

Aux doses employées la Lomidine n'a donné lieu en aucun cas à des symptômes d'intolérance.

\section{Conclusions sur la Pentamidine}

Très bon piroplasmicide sur $B$. bigemina, à condition d'employer une dose d'au moins $1 \mathrm{mg} / \mathrm{kg}$, mais qui ne stérilise pas les animaux de l'infection. Apparemment sans effet sur $B$. argentina aux doses indiquées par le fabriquant (à une exception près, le zébu normal traité à $5,8 \mathrm{mg} / \mathrm{kg}$, dose élevée d'ailleurs); il pourrait donc remplacer au laboratoire le trypanbleu, lorsqu'une sortie de $B$. bigemina doit être supprimée, sans affecter $B$. argentina, à faible dose, par exemple $2 \mathrm{mg} / \mathrm{kg}$; il a l'avantage d'une action plus régulière sur $B$. bigemina par rapport au trypanbleu, l'administration est commode (voie intramusculaire), et il n'y a pas d'intolérance. Aucune action sur les anaplasmes.

La Pentamidine semble avoir peu d'intérêt dans les circonstances pratiques, où le diagnostic différentiel entre les deux babésioses est le plus souvent impossible sans l'appui du microscope.

\section{ACETURATE DE DIMINAZINE ( $=$ Berenil, N.D. = Ganaseg, N.D.)}

Les premières expériences avec ce produit sur les Babesiae bovines semblent avoir été rapportées en 1955 par NEITZ (in: BAUER, 1955), expériences faites sur $B$. bigemina et $B$. argentina (nous considérons le parasite 
appelé $B$. bovis en Afrique du Sud comme $B$. argentina). Plusieurs auteurs ont enregistré de bons résultats par la suite. Nous ne donnerons pas tous les détails de nos expériences, son efficacité étant suffisamment connue, surtout en ce qui concerne $B$. bigemina.

Nos essais ont été faits avec le Berenil sous forme de granules, dissout peu avant l'emploi dans de l'eau stérile en solution à 7 p. 100, administré par la voie intramusculaire.

\section{B. BIGEMINA}

\section{Pouvoir curatif}

a) Animaux splénectomisés, traités lors du premier accès après primo-infection par la seringue.

Trois animaux, traités à $0,6,0,7$ et $3,0 \mathrm{mg} / \mathrm{kg}$, ont rapidement guéri. Les parasites, nombreux dans tous les cas, avaient beaucoup diminué en nombre en moins de $24 \mathrm{~h}$. et étaient alors dégénêrés; dans les deux cas le sang était négatif en moins de $48 \mathrm{~h}$., dans un cas $(0,7 \mathrm{mg} / \mathrm{kg})$ en moins de 3 jours. Dans tous les cas la température était encore normale au moment du traitement. L'animal traité à $0,6 \mathrm{mg} / \mathrm{kg}$ présentait une hémoglobinurie au moment du traitement, hémoglobinurie qui disparut 2 jours plus tard. Une rechute très importante, nécessitant un traitement 17 jours après le premier s'est produite, dans un cas, chez l'animal traité à $0,6 \mathrm{mg} / \mathrm{kg}$.

Un quatrième bovin, traité à $1,0 \mathrm{mg} / \mathrm{kg}$, est mort de piroplasmose $3 \mathrm{~h}$. plus tard. Il présentait une hémoglobinurie, une température quelque peu basse $\left(37,1^{\circ}\right)$ et une parasitémie très importante. Le traitement était donc trop tardif (bien qu'il ait été fait le premier jour où la parasitémie a atteint le degré +++ ; ajoutons que l'accès de piroplasmose a probablement été aggravé par d'importants accès concomitants à Theileria mutans (THEILER, 1907 ) et Eperythrozoon tuomii, UILENBERG, 1967).

b) Animal splénectomisé, traité lors d'une rechute secondaire importante.

De rares parasites dégénérés persistent le lendemain de l'administration de $4,0 \mathrm{mg} / \mathrm{kg}$, le sang est ensuite négatif.

\section{Pouvoir stérilisant}

Les parasites ont fait leur réapparition après usage de doses de 0,6 et $0,7 \mathrm{mg} / \mathrm{kg}$; dans les autres cas il n'a pas été possible de vérifier leur persistance ou non. Incité par le rapport de BARNETT (1965), qui signale un cas de stérilisation après $5 \mathrm{mg} / \mathrm{kg}$ et également par le cas enregistré auparavant (UILENBERG, 1965) d'un animal en apparence stérilisé par $3 \mathrm{mg} / \mathrm{kg}\left({ }^{*}\right)$, quelques expériences sur le pouvoir stérilisant du Berenil ont été faites.

Cas A. - Animal normal, inoculé par la seringue. Très faible parasitémie. Traitement à $2,4 \mathrm{mg} / \mathrm{kg}$. La splénectomie 16 jours plus tard montre que le sujet n'est plus porteur de $B$. Bigemina, et l'inoculation de la même souche, 22 jours après la splénectomie, lui cause une piroplasmose mortelle.

Cas B. - Animal splénectomisé, porteur de deux souches de $B$. bigemina (l'une sortie après la splénectomie, un mois et demi auparavant, l'autre transmise par la seringue 2 semaines auparavant). Très faible parasitémie. Traitement à $5,0 \mathrm{mg} / \mathrm{kg}$. Les parasites ne réapparaissent pas pendant les 3 ans et demi qui suivent et son sang ne transmet pas le parasite à dautres animaux indemnes pendant cette période. Pour exclure la possibilité d'une piroplasmose exclusivement cérébrale (en pensant au cas rapporté en 1965), une biopsie du cerveau est faite 5 mois après le traitement, mais aucune Baberia n'est trouvée dans le cortex cérébral. (La technique de JOHNSTON et CALLOW (1963), avec de légères modifications, a été suivie pour la biopsie.)

Cas C. - Animal splénectomisé, porteur d'une souche sortie après la splénectomie un mois et demi auparavant. Très faible parasitémie. Traitement à $5,0 \mathrm{mg} / \mathrm{kg}$. Les parasites ne réapparaissent pas pendant les 3 ans qui suivent, son sang ne les transmet pas pendant cette période à d'autres bovins indemnes, et une biopsie du cortex cérébral 5 mois après le traitement donne un résultat négatif. L'inoculation d'une autre souche de $B$. bigemina, 3 ans après le traitement, lui cause une piroplasmose grave, guérie par traitement.

Cas D. - Animal splénectomisé, porteur de deux souches de $B$. bigemina (l'une sortie après la splénectomie, un mois auparavant, l'autre

(:) Le sang de cet animal ne transmettait plus B. bigemma par la suite, et le parasite n'apparaissait pas dans son sang après plusieurs inoculations; toutefois, à ]'autopsie il existait une piroplasmose cérébrale. 
transmise par la seringue 2 semaines auparavant). Traitement à $5,0 \mathrm{mg} / \mathrm{kg}$. Parasitémie très faible. Les parasites ne sont pas observés pendant les 19 jours qui suivent, et son sang ne les transmet pas à un bovin splénectomisé indemne ( $100 \mathrm{ml}$, prélevés 19 jours après le traitement, inoculés par la voie intraveineuse). Il est abattu. De nombreuses $B$. bigemina se trouvent dans les capillaires du cortex cérébral, mais aucune dans le sang du cour et de loreille, ni sur calques et frottis du foie, du rein et du poumon.

Cas E. - Animal normal, inoculé par la seringue. Parasitémie assez faible. Traitement à $6,7 \mathrm{mg} / \mathrm{kg}$. La splénectomie 3 semaines plus tard montre que l'animal n'est plus porteur, et l'inoculation de la même souche, 26 jours après la splénectomie, lui cause une piroplasmose grave, guérie par traitement.

Le Berenil à $2,4 \mathrm{mg} / \mathrm{kg}$ peut donc stériliser les animaux de $B$. bigemina, mais la stérilisation nंest pas toujours obtenue même à $5,0 \mathrm{mg} / \mathrm{kg}$ (bien que l'animal puisse sembler stérilisé si le cortex cérébral n'est pas examiné). L'explication pour les cas rapportés pour BARNETT (1965), de deux animaux traités à 2,5 et $3,5 \mathrm{mg} / \mathrm{kg}$, qui ne montraient pas de parasites pendant environ 3 mois, mais résistaient néanmoins à une nouvelle inoculation, sans parasitémie, réside peut-être dans cette persistance des parasites dans le cerveau.

\section{B. ARGENTINA}

a) Animaux splénectomisés, traités lors du premier accès après primo-infection par la seringue.

Cinq cas ont répondu d'une façon relativement satisfaisante à des doses de 2,5 - 4,0 $4,9-5,0$ et $5,6 \mathrm{mg} / \mathrm{kg}$. Dans un cas l'hyperthermie avait disparu en moins de $24 \mathrm{~h}$., dans les autres une hyperthermie diminuée persistait le lendemain du traitement, mais la température était normale après $48 \mathrm{~h}$. Dans deux cas $(4,9$ et $5,0 \mathrm{mg} / \mathrm{kg}$ ) le sang était négatif le lendemain du traitement, dans les autres cas il ne l'était qu'après $48 \mathrm{~h}$. Un des animaux $(4,0 \mathrm{mg} / \mathrm{kg})$ a fait une rechute mortelle 49 jours après le traitement.

$6,1 \mathrm{mg} / \mathrm{kg}$ ont bien fait disparaître les parasites chez un sixième sujet, mais il a fallu 3 jours pour que la température redevienne à la normale.

Finalement, la parasitémie chez un septième sujet traité à $3,4 \mathrm{mg} / \mathrm{kg}$ augmentait le lendemain et persistait, inchangée, au deuxième jour après l'intervention; il a alors reçu un autre piroplasmicide. La température était de $40,0^{\circ}$ le jour du traitement, de $39,7^{\circ}$ le lendemain, et de $39,2^{\circ}$ le jour suivant.

b) Animaux splénectomisés, traités lors du premier accès après primo-infection par les tiques.

\begin{tabular}{|c|c|c|c|c|c|}
\hline & $\begin{array}{l}\text { Jours après } \\
\text { le traitement }\end{array}$ & Dose & Parasitémie & Tempéarture & Remarques \\
\hline Cas $A$ & $\begin{array}{l}0 \\
1 \\
2 \\
3\end{array}$ & $\begin{array}{l}5.2 \mathrm{mg} / \mathrm{kg} \\
9,7 \mathrm{mg} / \mathrm{kg}\end{array}$ & $\begin{array}{c}(+) \\
\text { rares } \\
(+) \\
\text { très rares } \\
\quad(+) \\
\text { très rares } \\
(+) \\
\text { très ráres }\end{array}$ & $\begin{array}{l}41 \div 0 \\
40 \% 2 \\
3905 \\
40 \% 0\end{array}$ & $\begin{array}{l}\text { Premier jour de l'accès parasitaire. } 2^{\mathrm{e}} \mathrm{de} \\
\text { laccès thermique. } \\
\text { Reçott un autre pıroplasmicide. }\end{array}$ \\
\hline Cas B & $\begin{array}{l}0 \\
1 \\
2 \\
3 \\
4\end{array}$ & $6,5 \mathrm{mg} / \mathrm{kg}$ & $\begin{array}{c}+ \\
+ \\
(+) \\
\text { très rares } \\
0 \\
(+) \\
\text { très rares } \\
0\end{array}$ & $\begin{array}{c}40 \times 0 \\
\text { normale } \\
» \\
»\end{array}$ & $\begin{array}{l}\text { Premier jour de l'accès parasitaire et } \\
\text { thermique. }\end{array}$ \\
\hline
\end{tabular}




\section{Pouvoir stérilisant}

Apparemment aucun aux doses employées: dans quatre des cas relatés, il a été possible de prouver la persistance dans l'organisme (après des doses de $2,5-3,4-4,0$ et $5,0 \mathrm{mg} / \mathrm{kg}$ ), soit par la réapparition des parasites dans le sang, soit par leur persistance dans le cerveau (examen de frottis du cortex cérébral en fin d'expériences).

De plus, trois expériences spéciales ont été faites :

Cas A et B : Deux porteurs splénectomisés, traités à $5,0 \mathrm{mg} / \mathrm{kg}$. Les parasites réapparaissent plus tard dans leur sang, et celui-ci transmet la maladie à d'autres bovins indemnes.

Cas C: Porteur splénectomisé, traité à $10,6 \mathrm{mg} / \mathrm{kg}$. Des $B$. argentina sont trouvếes dans le cortex cérébral à l'abattage en fin d'expérience, un mois et demi plus tard.

\section{A. MARGINALE ET A. CENTRALE}

A notre connaissance aucun auteur n'a rapporté une action du Berenil sur les anaplasmes et nous avons pu constater que l'administration de $5,1 \mathrm{mg} / \mathrm{kg}$, administré à un animal 7 jours après sa splénectomie, au $3^{\circ}$ jour de la rechute postopératoire à $A$. marginale, n'a aucunement empêché la multiplication des anaplasmes pendant les jours suivants.

\section{Toxicité}

Dans un cas, l'administration par voie intramusculaire de $4,9 \mathrm{mg} / \mathrm{kg}$ a causé une enflure relativement peu importante au point d'inoculation. L'enflure était quelque peu douloureuse et a persisté pendant 3 jours. Tout est ensuite rentré dans l'ordre.

Dans un autre cas, l'administration par voie intramusculaire de $10,0 \mathrm{mg} / \mathrm{kg}$ a été suivie d'une enflure locale très importante, l'animal ne pouvant plus marcher jusqu'à sa mort (dont elle semble la seule cause possible), 22 jours plus tard. Les muscles où l'injection avait été faite (muscles fessiers) avaient un aspect "cuit» et ils étaient infiltrés de liquide.

Dans tous les autres cas (même celui traité à $10,6 \mathrm{mg} / \mathrm{kg}$ ) aucun symptôme d'intolérance n'a été observé.

\section{Conclusions sur le Berenil}

Très bon piroplasmicide sur $B$. bigemina, même à des doses inférieures à $1 \mathrm{mg} / \mathrm{kg}$. Peut stériliser les animaux de ce parasite aux doses normales, indiquées par le fabriquant $(3,5 \mathrm{mg} / \mathrm{kg})$, mais la stérilisation n'est pas toujours obtenue même à $5 \mathrm{mg} / \mathrm{kg}$. L'action sur $B$. argentina est certaine, mais elle semble plus irrégulière et il faut des doses plus élevées. Les animaux ne sont pas stérilisés de cette infection par des doses allant jusqu'à $10,6 \mathrm{mg} / \mathrm{kg}$.

Pas d'action sur les anaplasmes.

La commodité d'administration (voie intramusculaire) et l'absence de toxicité aux doses normales, associées à son activité sur les deux babésioses, en font un piroplasmicide de valeur dans les circonstances pratiques.

\section{AMICARBALIDE \\ (= Pirodia, N.D., etc.)}

ASHLEY et al. (1960) ont rapporté l'activitê de ce produit dans la piroplasmose bovine à $B$. divergens (M'FADYEAN et STOCKMAN, 1911). KEMRON et al. (1960) signalent son activité sur $B$. argentina $(=B$. berbera), tout au moins au début de la maladie. SHONE et al. (1961) obtiennent de bons résultats sur $B$. bigemina, et signalent l'absence d'activité sur A. marginale. Plusieurs auteurs ont confirmé les résultats signalés par les chercheurs cités, contre les babésioses bovines.

Puisqu'il s'agit d'un produit récent, nous donnerons la plus grande partie des détails de nos expériences. Elles ont été faites avec la présentation «Pirodia », contenant 20 p. 100 d'amicarbalide; l'administration a été faite par la voie intramusculaire ou sous-cutanée (qui nous ont donné des résultats semblables).

\section{B. BIGEMINA}

\section{Pouvoir curatif}

a) Animaux splénectomisés, traités lors du premier accès après primo-infection par la seringue.

b) Animal splénectomisé, traité lors du premier accès après primo-infection par les tiques.

La dose de $2,9 \mathrm{mg} / \mathrm{kg}$ est sans effet apparent $24 \mathrm{~h}$. après l'administration; ni la parasitémie $(+$ à ++$)$, ni l'hyperthermie $\left(39,7^{\prime}\right)$ n'ont diminué. Un autre piroplasmicide est alors administré. 


\begin{tabular}{|c|c|c|c|c|c|}
\hline & $\begin{array}{c}\text { Jours après } \\
\text { le traitement }\end{array}$ & Dose & Parasitémie & Température & Remarques \\
\hline Cas $A$ & $\begin{array}{l}0 \\
1\end{array}$ & $1,1 \mathrm{mg} / \mathrm{kg}$ & 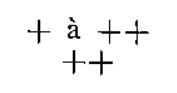 & $\underset{»}{\text { normale }}$ & Reçoit un autre piroplasmicide. \\
\hline Cas B & $\begin{array}{r}0 \\
1 \\
2 \\
3 \\
7 \\
71\end{array}$ & $1,1 \mathrm{mg} / \mathrm{kg}$ & $\begin{array}{c}+ \\
+ \\
(+) \\
\text { très rares } \\
0 \\
(+) \\
++\end{array}$ & $\begin{array}{c}40 \circ 2 \\
39 \circ 0 \\
\text { normale } \\
\$ \\
\$ \\
39 \times 7\end{array}$ & Reçoit un autre piroplasmicide. \\
\hline Cas $\mathrm{C}$ & $\begin{array}{l}0 \\
1 \\
2\end{array}$ & $1,5 \mathrm{mg} / \mathrm{kg}$ & $\begin{array}{l}+\underset{\mathrm{à}}{(++} \\
\text { très rares } \\
0\end{array}$ & $\begin{array}{c}4000 \\
\text { normale } \\
»\end{array}$ & $\begin{array}{l}\text { Aspect des parasites normal. } \\
\text { Les parasites réapparaissent ultérieure- } \\
\text { ment. }\end{array}$ \\
\hline Cas $\mathbf{D}$ & $\begin{array}{c}0 \\
1 \\
2 \text { à } \\
\quad \\
5 \\
7\end{array}$ & $3,2 \mathrm{mg} / \mathrm{kg}$ & $\begin{array}{c}+++ \\
(+) \underset{a ̀}{+}+ \\
(+) \\
\text { rares } \\
0 \\
(+)\end{array}$ & $\begin{array}{c}3904 \\
39 \circ 4 \\
\text { normale } \\
\\
» \\
״\end{array}$ & $\begin{array}{l}\text { Hémoglobinuric. } \\
\text { L'observation cesse. }\end{array}$ \\
\hline Cas $\mathrm{E}$ & $\begin{array}{l}0 \\
1 \\
2\end{array}$ & $5,1 \mathrm{mg} / \mathrm{kg}$ & $\begin{array}{l}+++ \\
(+) \\
\text { rares } \\
0\end{array}$ & $\begin{array}{c}41 * 2 \\
\text { normale } \\
»\end{array}$ & $\begin{array}{l}\text { Hémoglobinurie. } \\
\text { Parasites dégénérés. Hémoglobinurie. } \\
\text { Réapparition ultérieure. }\end{array}$ \\
\hline Cas $\mathrm{F}$ & $\begin{array}{c}0 \\
1 \\
2 \\
3 \stackrel{\text { มे } 5}{6}\end{array}$ & $\begin{array}{l}5,3 \mathrm{mg} / \mathrm{kg} \\
5,3 \mathrm{mg} / \mathrm{kg}\end{array}$ & $\begin{array}{c}+++ \\
(+) \text { à }+ \\
(+) \\
\text { assez rares } \\
0\end{array}$ & $\begin{array}{c}\underset{\Downarrow}{\text { normale }} \\
35^{\circ 1} \\
\text { normale }\end{array}$ & $\begin{array}{l}\text { Hémoglobinurie. } \\
\text { Parasites plus ou moins dégénérés. Hé- } \\
\text { moglobinurie. } \\
\text { Parasites plus au moins dégénérés. Hé- } \\
\text { moglobinurie. } \\
\text { Mort d'anémie }\end{array}$ \\
\hline
\end{tabular}

c) Animaux splénectomisés, traités lors de rechutes secondaires importantes.

Quatre cas, traités avec des doses de 1,5 à $6,7 \mathrm{mg} / \mathrm{kg}$. Dans tous les cas la parasitémie était importante à très importante. Le sang était négatif sur tous après 1 ou 2 jours, et dans les deux cas où il y avait de l'hyperthermie (traités à 5,0 et $6,7 \mathrm{mg} / \mathrm{kg}$ ) la température était normale $24 \mathrm{~h}$. après le traitement. Les Babesiae ont fait leur réapparition ultérieurement sur deux des sujets, traités à 1,5 et $2,8 \mathrm{mg} / \mathrm{kg}$, tandis que l'animal ayant reçu $6,7 \mathrm{mg} / \mathrm{kg}$ n'en a plus montré pendant une période d'observation de 84 jours.

d) Animaux normaux, traités lors du premier accès après primo-infection par la seringue.
Quatorze sujets, traités avec des doses variant entre 2,9 et $18,2 \mathrm{mg} / \mathrm{kg}$. Dans huit cas le sang était négatif $24 \mathrm{~h}$. après le traitement (à $2,9-3,1-5,0-5,3-5,4-5,7-6,2$ et $10,0 \mathrm{mg} / \mathrm{kg}$ ), dans les autres cas (traités à 3,1 $3,1-3,6-5,1-9,5$ et $18,2 \mathrm{mg} / \mathrm{kg}$ ) de rares parasites dégénérés persistaient, mais avaient disparu 2 jours après le traitement. Dans les cinq cas où l'accès fut accompagné d'hyperthermie, la température était normale $24 \mathrm{~h}$. après le traitement. Dans les deux cas où il $\mathrm{y}$ avait hémoglobinurie, celle-ci avait disparu le lendemain du traitement.

L'évolution a été suivie sur un des animaux, traité à $6,2 \mathrm{mg} / \mathrm{kg}$, par frottis de sang et prise de température d'heure en heure, et le pourcen- 
tage d'érythrocytes infestés a été compté aussi bien que possible. Voici le résultat:

\begin{tabular}{|c|c|c|}
\hline $\begin{array}{c}\text { Nombre } \\
\text { d'heures } \\
\text { apès le } \\
\text { traitement }\end{array}$ & Température & $\begin{array}{c}\text { Pourcentage } \\
\text { de globules infestés }\end{array}$ \\
\hline 0 & $40^{\circ 03}$ & 13 à 14 p. 100 \\
1 & $40^{\circ} 3$ & 16 p. 100 \\
2 & 4004 & 16 p. 100 \\
3 & 4003 & 8 à 9 p. 100 \\
4 & 4004 & 9 p. 100 \\
5 & 4002 & 5 à 6 p. 100 \\
6 & $40^{\circ} 5$ & 5 p. 100 \\
7 & 4007 & 5 p. 100 \\
8 & $40^{\circ} 5$ & 3 à 4 p. 100 \\
22 & $38^{\circ} 4$ & 0 p. 100 \\
\hline
\end{tabular}

Les parasites commencent à dêgénérer à partir de la $5^{\mathrm{e}}$ heure après le traitement.

\section{Pouvoir stérilisant}

Dans plusieurs cas il a été possible d'observer que des doses allant jusqu'à $5,3 \mathrm{mg} / \mathrm{kg}$ n'ont pas stérilisé les animaux de l'infection, dans d'autres cas il n'a pas été possible de vérifier la persistance ou non des parasites. Un sujet traité à $5,3 \mathrm{mg} / \mathrm{kg}$ a été splénectomisé 2 mois plus tard; l'animal n'avait pas été stérilisé de son infection. Un sujet traité à $18,2 \mathrm{mg} / \mathrm{kg}$ a ćté splénectomisé 3 semaines plus tard; l'opération a montré que ce traitement avait stérilisé l’animal de son infection, et l'inoculation de la même souche le mois suivant lui a causé une piroplasmose mortelle. Finalement, une expé- rience spéciale a encore été faite: Animal normal, inoculé avec $B$. bigemina, traité à $12,5 \mathrm{mg} / \mathrm{kg}$ lorsque les parasites ont commencé à se montrer dans le sang, splénectomisé un mojs plus tard: $B$. bigemina n'a pas fait de sortie postopératoire et l'inoculation de la même souche lui a causé une piroplasmose grave, qui a dû être traitée.

Il a donc été prouvé que des doses de 12,5 et $18,2 \mathrm{mg} / \mathrm{kg}$ peuvent stériliser les animaux de l'infection, tandis que les doses inférieures à $5-6 \mathrm{mg} / \mathrm{kg}$ semblent normalement laisser persister l'état de prémunition. BARNETT (1965) trouve que des doses de 5 et $7,5 \mathrm{mg} / \mathrm{kg}$ ne stérilisent pas, contrairement à la dose de $10 \mathrm{mg} / \mathrm{kg}$. PIPANO (1966) signale que la dose de $5 \mathrm{mg} / \mathrm{kg}$, administrée pendant l'accès, a apparemment stérilisé un animal sur quatre dt l'infection; $5 \mathrm{mg} / \mathrm{kg}$ pendant la période d'incubation stérilise. Notons que le fabriquant indique des doses normales de 5 à $10 \mathrm{mg} / \mathrm{kg}$ (doses d'ailleurs certainement nécessaires dans les circonstances pratiques, voir l'activité sur $B$. argentina, ci-dessous); une stérilisation de I'infection à $B$. bigemina est donc possible, ce qui peut être un avantage ou un désavantage, selon les circonstances.

\section{B. ARGENTINA}

\section{Pouvoir curatif}

a) Animaux splénectomisés, traités lors du premier accès après primo-infection par la seringue.

\begin{tabular}{|c|c|c|c|c|c|}
\hline & $\begin{array}{c}\text { Jours après } \\
\text { le traitement }\end{array}$ & Dose & Parasitémie & Température & Remarques \\
\hline Cas $\mathrm{A}$ & $\begin{array}{l}0 \\
1 \\
2 \\
3 \\
4\end{array}$ & $7,3 \mathrm{mg} / \mathrm{kg}$ & $\begin{array}{c}(+) \\
\text { assez nombr. } \\
+ \\
+ \\
(+) \\
\text { très rares } \\
0\end{array}$ & $\begin{array}{c}40 \circ 1 \\
40 \circ 6 \\
39 \circ 2 \\
\text { normale } \\
»\end{array}$ & $\begin{array}{l}\text { Premier jour de l'accès parasitaire et } \\
\text { thermique. }\end{array}$ \\
\hline Cas B & $\begin{array}{l}0 \\
1 \\
2 \\
3\end{array}$ & $\begin{array}{l}10,4 \mathrm{mg} / \mathrm{kg} \\
20,8 \mathrm{mg} / \mathrm{kg}\end{array}$ & $\begin{array}{c}(+) \\
\text { très rares } \\
(+) \\
\text { très rares } \\
(+) \\
\text { très rares } \\
0\end{array}$ & $\begin{array}{c}4002 \\
3904 \\
3904 \\
\text { normale }\end{array}$ & $\begin{array}{l}\text { Premier ou } 2 \text { jour de l'accès parasitaire } \\
\text { et thermique. }\end{array}$ \\
\hline
\end{tabular}




\begin{tabular}{|c|c|c|c|c|c|}
\hline & $\begin{array}{l}\text { Jours après } \\
\text { le traitement }\end{array}$ & Dose & Parasitémie & Température & Remarques \\
\hline Cas $\mathrm{C}$ & $\begin{array}{l}0 \\
1 \\
2\end{array}$ & $15,5 \mathrm{mg} / \mathrm{kg}$ & $(+) \underset{0}{+}+$ & $\underset{>}{40 n 5}$ & $\begin{array}{l}2^{e} \text { jour de l'accès parasitaire et ther- } \\
\text { mique. }\end{array}$ \\
\hline Cas D & $\begin{array}{l}0 \\
1 \\
2 \\
3\end{array}$ & $23,0 \mathrm{mg} / \mathrm{kg}$ & $\begin{array}{c}(+) \\
\text { rares } \\
(+) \\
\text { très rares } \\
(+) \\
\text { très races } \\
0\end{array}$ & $\begin{array}{c}3909 \\
3907 \\
\text { normale } \\
\text { }\end{array}$ & $\begin{array}{l}\text { Premier jour de l'accès parasitaire, } 2^{\mathrm{e}} \text { de } \\
\text { l'accès thermıqu. }\end{array}$ \\
\hline
\end{tabular}

b) Animal splénectomisé, traité lors du premier accès après primo-infection par les tiques.

\begin{tabular}{|c|c|c|c|c|c|}
\hline & $\begin{array}{l}\text { Jours aprês } \\
\text { le traitement }\end{array}$ & Dose & Parasitémie & Température & Remarques \\
\hline Un cas & $\begin{array}{l}0 \\
1 \\
2\end{array}$ & $\begin{array}{l}10,0 \mathrm{mg} / \mathrm{kg} \\
10,0 \mathrm{mg} / \mathrm{kg}\end{array}$ & $\begin{array}{c}(+) \\
\text { très rares } \\
(+) \\
\text { très rares } \\
0\end{array}$ & $\begin{array}{c}40 \cdot 3 \\
40 * 1 \\
\text { normale }\end{array}$ & $\begin{array}{l}4^{\prime 2} \text { jour de l'accès parasitaire, } 5^{\circ} \text { de l'ac- } \\
\text { cès thermique. }\end{array}$ \\
\hline
\end{tabular}

c) Animaux splénectomisés, traités lors de rechutes secondaires importantes.

\begin{tabular}{|c|c|c|c|c|c|}
\hline & $\begin{array}{l}\text { Jours après } \\
\text { le traitement }\end{array}$ & Dose & Parasitémue & Température & Remarques \\
\hline Cas $\mathrm{A}$ & $\begin{array}{l}0 \\
1 \\
2\end{array}$ & $10,6 \mathrm{mg} / \mathrm{kg}$ & $\begin{array}{c}(+) \\
\text { très rares } \\
(+) \\
\text { pas rares } \\
0\end{array}$ & $\begin{array}{c}3908 \\
3907 \\
\text { normale }\end{array}$ & 5* jour de la rechute parasitaire. \\
\hline Cas B & $\begin{array}{l}0 \\
1 \\
2 \\
3\end{array}$ & $13,0 \mathrm{mg} / \mathrm{kg}$ & $\begin{array}{c}+++ \\
(+) \\
\text { pas rares } \\
(+) \\
\text { très rares } \\
0\end{array}$ & $\begin{array}{c}\text { normale } \\
36^{\circ 0} \\
\text { normale } \\
\quad »\end{array}$ & $4^{e}$ jour de la rechute parasitaire. \\
\hline
\end{tabular}

d) Animal porteur, traité lors de la rechute postopératoire après la splénectomie.

\begin{tabular}{|c|c|c|c|c|c|}
\hline & $\begin{array}{c}\text { Jours après } \\
\text { le traitement }\end{array}$ & Dose & Parasitémie & Température & Remarques \\
\hline Un cas & 0 & $10,2 \mathrm{mg} / \mathrm{kg}$ & +++ & 3909 & $\begin{array}{l}\text { Taurin adulte, } \\
\text { 2c jour de la rechute parasitatire, premser } \\
\text { de l'hyperthermie. } \\
\text { Symptômes nerveux (*) } \\
\text { Plus de symptômes nerveux. } \\
1\end{array}$ \\
2
\end{tabular}

(*) Position anormale, chancèle, reste avec une touffe d'herbe dans la bouche sans bouger. 
e) Animaux normaux, traités lors du premier accès après primo-infection par la seringue.

Une dose de $9,5 \mathrm{mg} / \mathrm{kg}$, administrée à un veau frison au premier jour de l'accès thermique, avant que les parasites ne fussent décelés dans le sang, n'a pas empêché leur apparition le lendemain, tandis que la fièvre persistait, mais plus faible; le surlendemain il n'y avait plus ni parasitémie, ni hyperthermie.

Deux autres veaux frisons, traités à 15,0 et $15,4 \mathrm{mg} / \mathrm{kg}$, ont encore montré des parasites le lendemain de l'intervention et l'hyperthermie persistait; le surlendemain le sang était négatif, mais un des veaux présentait encore de l'hyperthermie, qui n'avait disparue que 3 jours après le traitement.

Finalement, un jeune zébu présentant de très rares parasites et une faible hyperthermie $\left(39,2^{\circ}\right)$ fut traité à $18,9 \mathrm{mg} / \mathrm{kg}$. Le lendemain il n'y avait plus parasitémie ni hyperthermie.

f) Animal normal, traité lors de l'accès après transmission par les tiques (cas naturel).

Adulte métis brahman-zébu local. Présenté pour traitement avec des symptômes nerveux, de l'abattement, ictère et anémie. Température $39,9^{\circ}$. Faible parasitémie. Un traitement à environ $14 \mathrm{mg}$ de Pirodia par $\mathrm{kg}$ est institué. 24 h. plus tard les symptômes nerveux ont disparu, la température est normale, mais l'animal est apathique et anémié et la parasitémie a augmenté. Il reçoit alors une seconde injection de Pirodia, à la même dose. Néanmoins l'animal meurt 2 jours après ce dernier traitement, et les capillaires du cortex cérébral sont emplis d'érythrocytes infestés par $B$. argentina.

\section{Pouvoir stérilisant}

Dans la majorité des cas il n'a pas été possible de vérifier le pouvoir stérilisant. Dans quatre cas, mentionnés ci-dessus, les parasites ont fait leur réapparition dans le sang, après des doses de $9,5-10,2-10,6$ et $15,4 \mathrm{mg} / \mathrm{kg}$ (dans un de ces cas après l'administration de $10,2 \mathrm{mg} / \mathrm{kg} 2$ jours de suite). Le cas naturel, cité ci-dessus, présentait une babésiellose cérébrale 2 jours après un traitement consistant en deux injections de $14 \mathrm{mg} / \mathrm{kg}$. De plus, un autre animal, traité à deux piroplasmicides différents, dont l'un était le Pirodia à $11,2 \mathrm{mg} / \mathrm{kg}$, hébergeait encore le parasite par la suite. Aussi, il ne semble pas que le Pirodia stérilise les animaux aux doses normales ct même supérieures à la normale.

\section{A. MARGINALE ET A. CENTRALE}

A six reprises nous avons pu constater une régression rapide de la rechute à $A$. marginale après splénectomie, suivant l'administration de Pirodia; de même dans un cas de rechute secondaire à $A$. marginale et un à $A$. centrale. La régression était au moins aussi rapide que celle suivant le traitement aux tétracyclines (voir plus loin). Dans un autre cas de rechute après splénectomie la régression n'a toutefois pas eu lieu.

Afin de vérifier une éventuelle activité, trois animaux splénectomisés ont été traités au Pirodia lors de leur premier accès à $A$, marginale après primo-infection par la seringue: Dans deux cas, traités à 7,5 et $9,5 \mathrm{mg} / \mathrm{kg}$, la parasitémie a commencé à diminuer respectivement 7 et 6 jours après le traitement, sans hyperthermie. Mais le troisième cas n'a pas semblé influencé par le produit:

\begin{tabular}{|c|c|c|c|c|}
\hline $\begin{array}{c}\text { Jours après } \\
\text { le traitement }\end{array}$ & Dose & Parasitémie & Température & Remarques \\
\hline 0 & $10,2 \mathrm{mg} / \mathrm{kg}$ & $\begin{array}{c}(+) \\
\text { assez rares } \\
(+)\end{array}$ & normale & 5e jour de l'accès parasitaire. \\
$1-3$ & $10,2 \mathrm{mg} / \mathrm{kg}$ & $\begin{array}{c}\text { en augmentation } \\
+ \\
+\mathrm{à}++\end{array}$ & $39^{*}+5$ & $\begin{array}{c}\text { Un autre produit (oxytétracycline) } \\
\text { est administré. }\end{array}$ \\
\hline
\end{tabular}

En conclusion : l'amicarbalide n'a probablement pas d'activité sur les anaplasmes (le cas contraire serait d'ailleurs assez surprenant), mais de plus amples expériences sont nécessaires avant de tirer des conclusions fermes (en tenant également compte des résultats irrégu- 
liers obtenus avec les tétracyclines, plus loin, qui possèdent néanmoins une activité indéniable).

\section{Toxicité}

Aucun symptôme d'intoxication ou d'intolérance locale n'a été remarqué, et cela à toutes les doses employées.

\section{Conclusions sur l'A micarbalide}

Très bon piroplasmicide contre $B$. bigemina, ayant une certaine activité même à la faible dose de $1,1 \mathrm{mg} / \mathrm{kg}$, mais l'action est alors très lente. La disparition des parasites aux doses plus élevées, quoique en moyenne un peu plus lente que celle causée par certains autres piroplasmicides, a été bien plus rapide que celle rapportée par BARNETT (1965). Le produit peut stériliser les animaux à des doses supérieures à $10 \mathrm{mg} / \mathrm{kg}$, et d'après les expériences de BARNETT (1965) et de PIPANO (1966) la stérilisation peut parfois se produire aux doses normales indiquées par le fabriquant.

Le Pirodia a indubitablement une action sur $B$. argentina, mais elle est lente même aux doses élevées. (RIEK (1968) le considère comme le meilleur produit contre ce parasite.) Il ne semble pas stériliser les animaux de l'infection.

L'action sur les anaplasmes est douteuse. Le fait qu'une régression de la rechute après splénectomie ait suivi le traitement peut être une coïncidence (bien que cela se soit produit dans six cas sur sept), ces rechutes rétrogradant souvent spontanément. Dans les trois cas traités lors d'accès après primo-infection, l'action a tout au plus été lente dans deux cas, nulle dans lautre (justement la dose la plus élevée).

La facilité d'administration (voie souscutanée ou intramusculaire) et l'absence de toxicité, même aux doses élevées, associées à son activité sur les deux babésioses, en font un piroplasmicide de valeur dans la pratique. Malheureusement son prix élevé, tout au moins à Madagascar, limite le champ de son utilisation.

\section{OXYTETRACYCLINE (= Terramycine, N.D.)}

MILLER et al. (1952, cités par MILLER, 1956) semblent avoir été les premiers à rap- porter l'activité de ce produit sur $A$. marginale. De nombreux auteurs l'ont confirmé. A notre connaissance aucun auteur ne rapporte une activité sur les Babesiae.

Son activité ayant été déjà amplement décrite, nous nous limiterons à un résumé des résultats obtenus, en donnant quelques exemples surprenants.

Dans toutes les expériences nous avons utilisé des préparations commerciales de Terramycine, soit la forme "Solution Injectable» (à $50 \mathrm{mg} / \mathrm{ml}$, injectée par voie intramusculaire, rarement par voie intraveineuse), soit la forme «Suspension Retard» (contenant $25 \mathrm{mg} / \mathrm{ml}$, injectée par voie intramusculaire). Nous n'avons pu observer aucune différence entre les deux présentations, ni entre les voies d'administration, en ce qui concerne l'activité sur les anaplasmes.

\section{B. BIGEMINA ET B. ARGENTINA}

Aucune expérience spéciale n'a été faite, mais de nombreuses observations par coïncidence ont montré que la Terramycine n'a aucune activité sur les Babesiae.

\section{A. MARGINALE ET A. CENTRALE}

\section{Pouvoir curatif}

a) Animaux splénectomisés, traités lors du premier accès après primo-infection par la seringue.

Cinq animaux avec $A$. marginale, quatre avec $A$. centrale. L'action du produit est extrêmement lente. Dans aucun cas la parasitémie n'a augmenté après le traitement, qui variait de 3,2 à $12,3 \mathrm{mg} / \mathrm{kg}$, mais elle ne commence à diminuer qu'après une période variant de 1 à 8 jours. Le sang est devenu négatif dans quatre cas seulement, de 8 à 13 jours après le traitement (de 3,9 à $12,3 \mathrm{mg} / \mathrm{kg}$ ), dans les autres cas une faible parasitémie persistait. Des rechutes parasitaires, avec ou sans hyperthermie, suivent. Dans les cas où l'accès est accompagné d'hyperthermie, celle-ci cède rapidement au traitement (dans 1 ou 2 jours).

Voici quelques exemples : 


\begin{tabular}{|c|c|c|c|c|c|}
\hline & $\begin{array}{l}\text { Jours après } \\
\text { le traitement }\end{array}$ & Dose & Parasitémie & Température & Remarques \\
\hline $\begin{array}{l}\text { Cas A } \\
\text { (A. mar- } \\
\text { ginale })\end{array}$ & $\begin{array}{c}0 \\
1-3 \\
4 \\
9 \\
15 \\
18 \text { à } 21 \\
22\end{array}$ & $3,2 \mathrm{mg} / \mathrm{kg}$ & $\begin{array}{l}+ \\
++ \\
++ \\
++ \text { à } \\
\text { rares } \\
+ \\
++ \\
+\end{array}$ & \begin{tabular}{|c|} 
normale \\
$»$ \\
$»$ \\
$»$ \\
$y^{\prime}$ \\
hyperthermie \\
(jusqu'à $\left.40^{\circ} 0\right)$ \\
normale \\
\end{tabular} & $\begin{array}{l}3 e \text { jour de l'accès parasitaire. } \\
\text { Guérison spontanée. }\end{array}$ \\
\hline $\begin{array}{l}\text { Cas B } \\
\text { (A. mar- } \\
\text { ginale })\end{array}$ & $\begin{array}{r}0 \\
1 \\
2 \\
3 \\
8 \\
17\end{array}$ & $\begin{array}{l}10,0 \mathrm{mg} / \mathrm{kg} \\
10,0 \mathrm{mg} / \mathrm{kg}\end{array}$ & $\begin{array}{l}+ \\
+ \\
+ \\
(+) \stackrel{\text { à }}{+} \\
+ \\
+\end{array}$ & $\begin{array}{c}\text { normale } \\
\gg \\
39^{\circ} 2 \\
\text { normale } \\
\gg\end{array}$ & $\begin{array}{l}\text { 10e jour de l'accès parasitaire. } \\
\text { Guérison spontanée. }\end{array}$ \\
\hline $\begin{array}{l}\text { Cas C } \\
\text { (A. cen- } \\
\text { trale) }\end{array}$ & $\begin{array}{c}0 \\
1-2 \\
6 \\
7 \\
8 \\
13\end{array}$ & $6,2 \mathrm{mg} / \mathrm{kg}$ & $\begin{array}{c}+++ \\
+++ \\
++ \\
+ \\
(+) \\
0\end{array}$ & $\begin{array}{c}40^{\circ} 1 \\
\text { normale } \\
» \\
» \\
»\end{array}$ & $\begin{array}{l}11^{\mathrm{e}} \text { jour de l'accès parasitaire, } 2^{\mathrm{e}} \mathrm{de} \\
\text { l'accès thermique. } \\
\text { Des rechutes suivent. }\end{array}$ \\
\hline $\begin{array}{l}\text { Cas D } \\
\text { (A. cen- } \\
\text { trale) }\end{array}$ & $\begin{array}{c}0 \\
1-2 \\
3-4 \\
5 \\
10 \\
13 \\
17 \\
34\end{array}$ & $12,3 \mathrm{mg} / \mathrm{kg}$ & $\begin{array}{c}++\grave{a}+++ \\
+ \text { à }++ \\
(+) \grave{a}+ \\
(+) \\
(+) \text { rares } \\
0 \\
(+) \\
+-\end{array}$ & $\begin{array}{c}4000 \\
\text { normale } \\
» \\
» \\
» \\
» \\
»\end{array}$ & $\begin{array}{l}15 \mathrm{e} \text { jour de l'accès parasitaire, } 5 \mathrm{e} \text { de } \\
\text { l'accès thermique. } \\
\text { D'autres rechutes suivent. }\end{array}$ \\
\hline
\end{tabular}

b) Animaux normaux, traités lors du premier accès après primo-infection par la seringue.
Un animal infecté avec $A$. marginale, quatre avec $A$. centrale. La réponse est aussi lente que chez les animaux splénectomisés. Un exemple :

\begin{tabular}{|c|c|c|c|c|c|}
\hline & $\begin{array}{l}\text { Jours après } \\
\text { le traitement }\end{array}$ & Dose & Parasitémie & Température & Remarques \\
\hline $\begin{array}{l}\text { Cas } \\
\text { infecté } \\
\text { avec } \\
\text { A. margi- } \\
\text { nale }\end{array}$ & $\begin{array}{r}0 \\
1 \\
2 \\
3 \\
6 \\
10\end{array}$ & $5,1 \mathrm{mg} / \mathrm{kg}$ & $\begin{array}{c}+ \\
(+) \text { à }+ \\
(+) \text { à }+ \\
(+) \\
(+) \text { rares } \\
0\end{array}$ & $\begin{array}{c}3902 \\
39 \circ 1 \\
\text { normale } \\
» \\
» \\
»\end{array}$ & $\begin{array}{l}\text { 7e jour de l'accès parasitajre, premier } \\
\text { de j'accès thermique. }\end{array}$ \\
\hline
\end{tabular}

c) Animaux porteurs, traités lors de la première rechute après splénectomie.

Sept animaux avec $A$. marginale, un avec A. centrale, traités à 4,2 à $6,0 \mathrm{mg} / \mathrm{kg}$. Le traitement a été suivi sur tous par une lente diminution puis disparition (de 5 à 17 jours après le traitement) des anaplasmes. De nouvelles rechutes ont eu lieu sur tous les animaux qui ont pu être contrôlés pendant quelque temps, sauf sur un que nous ne pouvons pas expliquer : 


\begin{tabular}{|c|c|c|c|}
\hline & $\begin{array}{l}\text { Jours après } \\
\text { le traitement }\end{array}$ & Dose & Parasıtémie \\
\hline $\begin{array}{l}\text { Bovin } \\
\text { B } 73\end{array}$ & $\begin{array}{c}0 \\
1-2 \\
3 \\
8 \\
10-194\end{array}$ & $4,7 \mathrm{mg} / \mathrm{kg}$ & $\begin{array}{c}(+)^{+} \mathrm{à} \\
(+) \\
(+) \text { rares } \\
0\end{array}$ \\
\hline
\end{tabular}

Le sang reste négatif pendant toute cette période, malgré le fait que $B 73$ reçoit $150 \mathrm{ml}$ de sang (en i.v. et s.c.) de trois bovins porteurs chroniques au jour $77,50 \mathrm{ml}$ (en i.v.) d'un porteur chronique (dont le sang inoculé le même jour à un autre bovin splénectomisé transmet l'anaplasmose) au jour 125 , et $50 \mathrm{ml}$ de sang (en s.c.) d'un animal ayant une parasitémie d'un degré + , au jour 152. Ajoutons que $100 \mathrm{ml}$ de sang de $B 73$, prélevé 62 jours après son traitement, ne transmettent pas l'anaplasmose à un bovin splénectomisé indemne (inoculation par voie intraveineuse). Il s'agit d'A. marginale.

d) Animaux splénectomisés, traités lors de rechutes secondaires.

Trois animaux portant $A$. marginale, traités avec des doses de 5 à $8 \mathrm{mg} / \mathrm{kg}$, sont guéris après une disparition lente des anaplasmes. Des rechutes nouvelles ont suivi. Des conclusions ne sont guère possibles, étant donné que la guérison spontanée des rechutes est la règle. Mais l'accès sur un quatrième animal n'a pas cédé au traitement.

\section{Pouvoir stérilisant}

Apparemment aucun lors d'une administration unique, même avec des doses de 10,0 et $12,3 \mathrm{mg} / \mathrm{kg}$, en ne considérant pas le cas inexpliqué du bovin B 73. Il n'en est pas de même lors de l'administration prolongée de Terramycine, comme l'ont montré SPLITTER et MILLER (1953, cités par MILLER, 1956).

\section{Toxicité}

La Terramycine a été bien supportée à toutes les doses. Toutefois, une accélération passagère de la respiration est habituellement observée lors de l'administration par voie intraveineuse (solution injectable).

\section{Conclusions sur l'oxytétracycline}

Pas d'action sur les Babesiae. Une activité sur les anaplasmes est certaine, mais la diminution des parasites est extrêmement lente; dans un cas $10 \mathrm{mg} / \mathrm{kg}$ n'ont pas empêché une augmentation des anaplasmes. Les animaux ne sont pas stérilisés de l'infection. Il semble que l'action principale soit une inhibition de la multiplication des parasites et non une destruction. L'influence sur l'hyperthermie semble plus rapide. Dans les conditions pratiques, le traitement spécifique de l'anaplasmose est de toute façon souvent tardif (voir aussi MILLER, 1956), l'accès parasitaire et thermique ayant dépassé son cap lorsque les symptômes cliniques (anémie) sont observés; il est nécessaire de combiner le traitement spécifique avec un traitement symptomatique de l'anémie. Commode à administrer (vole intramusculaire, la voie intraveineuse ne semblant pas présenter d'avantages), et apparemment sans danger lors d'une administration unique.

\section{CHLORTETRACYCLINE (= Auréomycine)}

L'activité de l'Auréomycine sur l'anaplasmose étant suffisamment décrite depuis les premières expériences en 1952 (voir MILLER, 1956), nous ne donnerons pas tous les détails de nos essais.

L'Auréomycine a toujours été administrée par voie intraveineuse, en solution à 5 p. 100 , préparée juste avant l'emploi.

\section{B. BIGEMINA}

JANSEN (1953) ayant rapporté l'activité de l'Auréomycine sur Babesia equi (LAVERAN, 1901), parasite peu sensible à la plupart des piroplasmicides, nous avons fait une expérience sur $B$. bigemina, une des espèces les plus sensibles aux piroplasmicides.

L'administration de $9,9 \mathrm{mg} / \mathrm{kg}$ d'Auréomycine par voie intraveineuse n'a eu aucune influence sur la multiplication des $B$. bigemina lors du premier accès après primo-infection par la seringue d'un splénectomisé. La parasitémie, faible au moment de l'administration, a atteint le degré ++2 jours plus tard, en même temps que s'est déclarée une hyperthermie; un autre piroplasmicide a alors dû être administré.

\section{B. ARGENTINA}

Aucune expérience spéciale, mais des observations par coïncidence ont montré que l'Auréomycine n'a pas d'activité sur ce parasite. 


\section{A. MARGINALE ET A. CENTRALE}

\section{Pouvoir curatif}

a) Animaux splénectomisés, traités lors du premier accès après primo-infection.

Trois animaux avec $A$. marginale, dont deux infectés par la seringue et un par les tiques (bovin $\mathrm{n}^{\prime \prime} 1109$, voir UILENBERG, sous presse). Ce dernier animal, traité à $4,8 \mathrm{mg} / \mathrm{kg}$, a apparemment répondu au traitement: la parasitémie a commencé à baisser 5 jours plus tard, et les anaplasmes étaient rares au jour 7; une rechute a d'ailleurs suivi à partir du jour 8 . Dans les deux autres cas l'influence du traitement a été faible ou nulle; un de ces sujets est mort le lendemain du traitement (à $3,8 \mathrm{mg} / \mathrm{kg}$ ), par l'anémie due à l'anaplasmose (ajoutons qu'il était déjà au $11^{\mathrm{e}}$ jour de l'accès parasitaire); l'autre est donné en exemple ci-dessous :

\begin{tabular}{|c|c|c|c|c|}
\hline $\begin{array}{l}\text { Jours après } \\
\text { le traitement }\end{array}$ & Dose & Parasitémie & Température & Remarques \\
\hline $\begin{array}{l}0 \\
1 \\
2 \\
3 \\
4 \\
5 \\
6 \\
7 \\
8\end{array}$ & $\begin{array}{l}5,0 \mathrm{mg} / \mathrm{kg} \\
5,0 \mathrm{mg} / \mathrm{kg}\end{array}$ & $\begin{array}{l}++ \\
++ \\
+++ \\
+++ \\
+++ \\
+++ \\
+++ \\
++ \\
+\end{array}$ & $\begin{array}{c}3901 \\
3905 \\
4002 \\
3904 \\
40^{\prime \prime 5} \\
3906 \\
3906 \\
3908 \\
\text { normale }\end{array}$ & $\begin{array}{l}\text { 5e jour de l'accès parasitaire, premier } \\
\text { de l'accès thermique. } \\
\text { Reçoit } 10 \mathrm{mg} \text { de Terramycine par } \\
\mathrm{kg} \text { (i.m.) } \\
\text { Reçoit encore } 10 \mathrm{mg} / \mathrm{kg} \text { de Terra- } \\
\text { mycine (i.m.) }\end{array}$ \\
\hline
\end{tabular}

Le sang est négatif à partir du jour 10 , mais une rechute suit 10 jours plus tard.

b) Animaux traités lors de rechute postopératoire après splénectomie.

Six animaux avec $A$. marginale, traités à des doses de 3,8 à $13,0 \mathrm{mg} / \mathrm{kg}$. Sur quatre sujets le sang est devenu négatif de 5 à 9 jours après le traitement. Chez un autre animal on observait une diminution graduelle de la parasitémie dès le lendemain du traitement (à $5,7 \mathrm{mg} / \mathrm{kg}$ ), mais le sang ne devenait jamais entièrement négatif. Finalement, la parasitémie chez le $6^{*}$ sujet, traité à $5,5 \mathrm{mg} / \mathrm{kg}$, a augmenté d'assez faible au moment du traitement à un degré de ++ au jour 9, pour ensuite diminuer graduellement; le sang était négatif au jour 18. Des rechutes ont suivi les trois animaux qui ont pu être suivis pendant quelque temps.

\section{Pouvoir stérilisant}

Apparemment aucun aux doses normales. Il est connu que des doses administrées pendant une longue période peuvent stériliser (voir MILLER, 1956), et nous avons essayé de débarasser 2 porteurs splénectomisés d'A. centrale de l'infection en employant des tétracyclines.
Chaque animal a reçu une injection de Terramycine à $5,5 \mathrm{mg} / \mathrm{kg}$ par voie intramusculaire. A partir du lendemain on a administré de l'Auréomycine technique "per os », les animaux recevant $10 \mathrm{mg} / \mathrm{kg}$ quotidiennement pendant un mois. Le lendemain de la dernière administration ils ont encore reçu $5,5 \mathrm{mg}$ de Terramycine par $\mathrm{kg}$, par voie intramusculaire.

Les deux sujets se sont en effet avérés indemnes d' $A$. centrale par la suite : un animal a été observé pendant plus de 2 ans, son sang n'a jamais plus montré d'anaplasmes et ne les a pas non plus transmis à d'autres animaux splénectomisés. L'autre, observé pendant 11 mois, n'en a pas révélé non plus, et son sang s'est avéré non infectieux pour d'autres animaux splénectomisés pendant cette période.

\section{Toxicité}

L'Auréomycine a été, à une exception près, bien tolérée aux doses employées, administrées par voie intraveineuse, l'injection étant faite lentement. Un veau frison, inoculé avec $10 \mathrm{mg} / \mathrm{kg}$, est mort par choc dans la demiheure suivante; à l'autopsie on trouvait un odème pulmonaire sanguinolent.

Nous avons également injecté à trois bovins l'Auréomycine en solution à 5 p. 100 par la 
voie intramusculaire, afin d'observer une réaction locale éventuelle (de 10 à $30 \mathrm{ml}$ dans les muscles fessiers à des sujets pesant environ 100 à $250 \mathrm{~kg}$ ). Une enflure relativement peu importante s'est développée, persistant pendant plus d'une semaine, sans boiterie importante; ensuite tout est rentré dans l'ordre. Nous ne savons pas si cette méthode d'administration a la même efficacité du point de vue thérapeutique que la voie normale, intraveineuse.

\section{Conclusions sur l'Auréomycine}

Sans action sur les Babesiae. Son action sur les anaplasmes est certaine, étant donné qu'elle peut stériliser les animaux de l'infection, mais elle semble encore plus lente, aux mêmes doses, qu'avec la Terramycine.

Elle présente l'inconvénient de devoir être administrée par la voie intraveineuse.

\section{SPIROTRYPAN (N.D.)}

Ce produit relativement récent, un arsénobenzol soufré, a été commercialisé pour la chimiothérapie de l'anaplasmose. ZUBIATE (1956), VOGELSANG et PIERETTI (1957) et SEIFERT (1960) par exemple rapportent de bons résultats.

Nos expériences ont été faites avec la solution commerciale Spirotrypan « fort ", contenant d'après le fabriquant 4 p. 100 d'arsenic et 0,86 p. 100 de soufre organique. La dose préconisée est de 20 à $40 \mathrm{ml}$ pour les bovins, administrés par voie intraveineuse, donc environ $0,1 \mathrm{ml} / \mathrm{kg}$, dose que nous avons presque toujours dépassée. Nous ne donnerons pas tous les détails de nos observations, qui semblent assez concluantes.

\section{B. BIGEMINA ET B. ARGENTINA}

Aucune activité (observations par coïncidence, et lors d'une expérience spéciale où l'injection de $20 \mathrm{ml}$ à un bovin splénectomisé de $170 \mathrm{~kg}$ n'empêchait nullement la multiplication de $B$. bigemina pendant le premier accès après primo-infection).

\section{A. MARGINALE ET A. CENTRALE}

a) Animaux splénectomisés, traités lors du premier accès après primo-infection par la seringue.

Trois animaux infestés avec $A$, marginale ont été traités avec des doses de 0,21 à $0,25 \mathrm{ml} / \mathrm{kg}$, de 9 à 15 jours après le début de l'accès parasitaire, sans hyperthermie dans deux cas, une température de $39,2^{\circ}$ dans l'autre. La parasitémie a commencé à diminuer 4 à 5 jours plus tard, avec toutefois une augmentation pendant 2 jours après le traitement dans un des cas. La diminution était aussi lente qu'après les traitements aux tétracyclines. Dans l'unique cas où une légère hyperthermie accompagnait l'accès, la température était normale le lendemain du traitement.

Une conclusion n'est guère possible, étant donné que les rémissions spontanées sont fréquentes, et qu'aucun des sujets n'a été traité au début de l'accès.

Un sujet infecté avec $A$. centrale fut traité à $0,25 \mathrm{ml} / \mathrm{kg}$, sans influence apparente :

\begin{tabular}{|c|c|c|c|}
\hline $\begin{array}{l}\text { Jour après } \\
\text { le traitement }\end{array}$ & Parasitémie & Température & Remarques \\
\hline $\begin{array}{l}0 \\
1 \\
2 \\
3 \\
4 \\
5 \\
5 \\
6 \text { à } 17 \\
18\end{array}$ & $\begin{array}{c}++ \\
++ \\
++ \\
++ \\
++\stackrel{a}{+}+++ \\
+\stackrel{a}{+}++ \\
\text { en diminution } \\
0\end{array}$ & $\begin{array}{c}40^{\circ} 0 \\
40^{\circ} 0 \\
39^{\circ} 4 \\
39 \circ 6 \\
40^{\circ} 0 \\
39^{\circ} 0 \\
\text { normale } \\
»\end{array}$ & $\begin{array}{l}10 \text { jour de l'accès parasitaıre, premier de l'hyperthermie. } \\
\text { Reçott de la Terramycine }(12,0 \mathrm{mg} / \mathrm{kg} \text {, en i.m. }) \text {. }\end{array}$ \\
\hline
\end{tabular}

b) Animaux traités lors de la rechute postopératoire après splénectomie.

Quatre cas infectés avec $A$. marginale, un avec $A$. centrale, traités avec 0,20 à $0,28 \mathrm{ml} / \mathrm{kg}$.
Le traitement n'a pas semblé influencer l'évolution des rechutes:

Dans un seul cas il n'y avait pas d'augmentation du niveau de la parasitémie après le 
traitement, mais elle persistait au même niveau $(++$ à -+++$)$ pendant 3 jours; une diminution graduelle suit, et le sang est négatif 13 jours après le traitement. Dans les quatre autres cas le nombre de parasites augmentait pendant 1 à 2 jours après le traitement, pour diminuer graduellement ensuite sur un sujet, tandis que sur un deuxième ils persistaient au même niveau encore pendant 5 jours avant de diminuer. Les deux autres sujets sont morts: augmentation de la parasitémie le lendemain du traitement sur l'un, ensuite taux stable (degré ++ ) pendant 4 jours malgré le fait qu'il a reçu une deuxième injection de Spirotrypan à la même dose 2 jours après la première; l'animal est mort d'anaplasmose 4 jours après le premier traitement, avec lésions intenses d'anémie. Augmentation pendant les 2 jours suivant le traitement sur l'autre; la parasitémie atteint le degré + au $3^{e}$ jour; il reçoit alors une seconde injection de Spirotrypan (comme la première à $0,25 \mathrm{ml} / \mathrm{kg}$ ), mais il meurt le lendemain de façon inexpliquée, car aucune lésion spécifique n'est relevée à l'autopsie. Intoxication par le Spirotrypan?

c) Animal splénectomisé, traité lors de rechutes secondaires importantes.

Un cas, traité à $0,20 \mathrm{ml} / \mathrm{kg}$. Degré de parasitémie + à ++ . Elle se maintient pendant 10 jours au degré +; l'animal reçoit alors de la Terramycine. Le même sujet est encore traité à $0,1 \mathrm{ml}$ de Spirotrypan par $\mathrm{kg}$ lors d'une autre rechute; degré de parasitémie +; elle se maintient au degré (+) à + pendant les 41 jours qui suivent, dassez nombreux parasites persistent ensuite encore pendant 25 jours, et l'animal est alors traité à la Terramycine.

d) Animal normal, traité lors d'une rechute secondaire importante.

Un cas, traité à $0,18 \mathrm{ml} / \mathrm{kg}$. Parasitémie ++ . Elle se maintient au même niveau pendant les 4 jours qui suivent, d'assez nombreux parasites persistent encore pendant au moins 20 jours après le traitement.

\section{Toxicité}

L'injection (faite lentement) a toujours été bien supportée. Il est possible que l'animal mort le lendemain du second traitement à $0,25 \mathrm{ml} / \mathrm{kg}, 3$ jours après le premier, soit mort d'intoxication par le Spirotrypan.

\section{Conclusions sur le Spirotrypan}

Aucune action sur les Babesiae. Semble également dépourvu d'action sur les anaplasmes. Le produit a en outre l'inconvénient de devoir être administré par voie intraveineuse.

\section{SPIRAMYCINE (= Suanovil, N.D.)}

Aucun auteur ne rapporte, à notre connaissance, une activité de cet antibiotique sur les parasites qui nous intéressent. Nous avons fait deux expériences sur les anaplasmes.

Le produit a été administré en solution à 5 p. 100, par voie intramusculaire (marque Suanovil), à deux animaux lors de la rechute postopératoire à $A$. marginale après splénectomie.

Cas A : Dose $36 \mathrm{mg} / \mathrm{kg}$; parasitémie +++ ; pas d'hyperthermie. La parasitémie persiste au niveau +++ pendant 3 jours après le traitement, l'animal est trouvé mort par l'anémie due à lanaplasmose au $4^{\mathrm{e}}$ jour.

Cas B : Dose $64 \mathrm{mg} / \mathrm{kg}$; parasitémie ++ ; pas d'hyperthermie. La parasitémie augmente pour atteindre le degré +++3 jours après le traitement; ensuite diminution graduelle et les parasites sont très rares 7 jours après le traitement; l'observation cesse le lendemain.

\section{Conclusions}

Apparemment pas d'activité sur les anaplasmes. Le produit a été bien supporté.

\section{NEOARSPHENAMINE (= Novarsénobenzol, N.D.)}

Certains auteurs (cités par CURASSON, 1943) rapportent de bons résultats sur des Babesiae et les anaplasmes.

Une expérience a êté faite sur $B$. bigemina, une autre sur $A$. marginale, employant une solution à 10 p. 100 , préparée extemporairement, injectée par voie intraveineuse.

\section{B. BIGEMINA}

La dose de $12,5 \mathrm{mg} / \mathrm{kg}$ n'a eu aucune influence sur la multiplication des parasites pendant le premier accès d'un splénectomisé, 
infecté par la seringue. La parasitémie, d'un degré $(+)$ a + au moment du traitement, a atteint le degré +++2 jours plus tard, tandis que la température, normale lors du traitement, était de $40,0^{\circ} 2$ jours plus tard, en même temps qu'une hémoglobinurie se développât. L'animal a été guéri par un autre piroplasmicide.

\section{A. MARGINALE}

La dose de $3,6 \mathrm{mg} / \mathrm{kg}$ n'a pas influencé sur la rechute postopératoire suivant la splénectomie. Les parasites, rares au moment du traitement, sont au degré +2 jours plus tard; l'animal reçoit alors de la Terramycine.

\section{Conclusions}

Le Novarsénobenzol n'a pas d'action sur les Babesiae (ainsi que l'ont encore confirmé quelques observations par coüncidence, également en ce qui concerne $B$. argentina). Aucune action non plus sur $A$. marginale à la dose (assez faible) de $3,6 \mathrm{mg} / \mathrm{kg}$. Il a l'inconvénient de devoir être injecté par voie intraveineuse.

\section{MEPACRINE DICHLORHYDRATE (= Quinacrine)}

Par curiosité, puisque le produit est actif dans le paludisme humain, nous avons fait un essai sur $B$. bigemina : Dose $6.7 \mathrm{mg} / \mathrm{kg}$, administré par voie intraveineuse en solution à 2,5 p. 100. Parasitémie + à ++ , température normale. Premier accès d'un splénectomisé après primo-infection par la seringue. Le lendemain la parasitémie est de +++ , la température de $40,0^{\circ}$, et une hémoglobinurie s'est déclarée. Le sujet reçoit alors un autre piroplasmicide.

\section{Conclusion}

Dépourvu d'activité sur B. bigemina.

\section{DISCUSSION ET CONCLUSIONS}

Dans certains cas aucun produit ne peut sauver l'animal de la mort lorsque l'évolution de la maladie est trop avancée et l'animal peut mourir de l'anémie causée par la piroplasmose vraie ou l'anaplasmose, malgré la destruction des parasites par le traitement; la babésiellose cérébrale semble également difficilement influençable. Le traitement doit donc être le plus précoce possible.

En résumant les résultats obtenus avec les différents produits expérimentés, nous arrivons. aux conclusions suivantes:

\begin{tabular}{|c|c|c|c|c|}
\hline & B. bigemina & B. argentina & $\begin{array}{l}\text { A. marginale } \\
\text { et } A \text {. centrale }\end{array}$ & $\begin{array}{l}\text { Inconvénients } \\
\text { d'administration } \\
\text { ou de toxicité }\end{array}$ \\
\hline $\begin{array}{l}\text { Trypanbleu } \\
\text { Euflavine } \\
\text { Sulfate de quinuronium } \\
\text { Pentamidine } \\
\text { Acéturate de dimınazine } \\
\text { Amicarbalide } \\
\text { Oxytétracycline } \\
\text { Chlortétracycline } \\
\text { Spirotrypan } \\
\text { Spiramycine } \\
\text { Néoarsphénamine } \\
\text { Mépacrine }\end{array}$ & $\begin{array}{l}++ \\
++ \\
+++ \\
+++ \\
+++ \\
++ \\
0 \\
0 \\
0 \\
\text { inconnu } \\
0 \\
0\end{array}$ & $\begin{array}{c}0 \\
+ \\
(+) \\
0 \\
+ \\
+ \\
0 \\
0 \\
0 \\
\text { inconnu } \\
0 \\
\text { inconnu }\end{array}$ & $\begin{array}{l}0 \\
0 \\
0 \\
0 \\
0 \\
0(?) \\
+ \\
(+) \\
0 \\
0 \\
0 \\
\text { inconsu }\end{array}$ & $\begin{array}{l}\text { oui } \\
\text { oui } \\
\text { oui } \\
- \\
\cdots- \\
- \\
- \\
\text { oui } \\
\text { oui } \\
\text { ou1 } \\
\text { oui }\end{array}$ \\
\hline
\end{tabular}

Tenant compte de l'activité sur les différents parasites, la voie d'administration et la toxicité, il nous semble qu'il faudrait retenir de préférence pour le traitement dans la pratique (lorsqu'on n'a pas l'occasion d'examiner un frottis de sang) l'acéturate de diminazine ou l'amicarbalide dans le cas d'une babésiose, l'oxytétracycline dans celui d'une anaplasmose; la combinaison de l'oxytétracycline avec un des deux piroplasmicides mentionnés lorsqu'on est incertain du diagnostic clinique.
Les doses à conseiller dans ces conditions nous semblent être, en tenant également compte des précisions fournies par les fabriquants, de 3,5 à $7 \mathrm{mg} / \mathrm{kg}$ pour l'acéturate de diminazine, de 5 à $10 \mathrm{mg} / \mathrm{kg}$ pour l'amicarbalide et l'oxytétracycline.

Le traitement de la piroplasmose, entrepris à temps, ne présente aucune difficulté, celui de la babésiellose et de l'anaplasmose n'est souvent pas satisfaisant. 


\title{
SUMMARY
}

\section{Notes on bovine babesiosis and anaplasmosis in Madagascar} III. Experiments in treatment

The author reports the results obtained in treatment of bovine babesiosis and anaplasmosis in Madagascar, using several products.

\section{RESUMEN}

\begin{abstract}
Notas sobre las babesiosis y la anaplasmosis de los bovinos en Madagascar III. Ensayos de tratamiento

El autor nota los resultados obtenidos en el tratamiento de las babesiosis y de la anaplasmosis de los bovinos en Madagascar, utilizando varios productos.
\end{abstract}

\section{BIBLIOGRAPHIE}

ANONYME, «Akiron. A new preparation for the treatment of piroplasmosis, babesiellosis, and nuttalliosis », J.S. Afr. vet. med. Ass., 1935, 6 : 54-56.

ASHLEY (J. N.), BERG (S.S.) et LUCAS (J.M.S.), \& 3 : 3'-diamidinocarbanilide: a new drug active against babesial infections », Nature, Lond., 1960, 185: 461 .

BARNETT (S.F.), «The chemotherapy of Babesia bigemina infection in cattle $»$, Res. vet. Sci., 1965, $6: 397-415$

BAUER (F.), «Trypanosomen- und Babesienerkran kungen in Afrika und ihre Behandlung mit dem neuen Präparat "Berenil" ", Z. Tropenmed. u. Parasit., 1955, 6: 129-140.

BECKMAN (H.) et SMITH (J.), «A new method of counting Plasmodia in avian malaria infections \&, J. Lab. clin. Med., 1943, 28 ; 1735-1740.

BUCK (G.), QUESNEL (J.J.) et RAMBELOSON (L.), « Traitement de l'anaplasmose bovine par la combinaison gonacrine-lomidine ", Rev. Elev. Méd. vét. Pays trop., 1951-1952, $5: 7$.

CALLOW (L. L.) et McGREGOR (W.), "Quinuronium sulphate poisoning of cattle $n$, Vet. Rec., 1967, 81: 175-176.

CALLOW (L. L.) et MELLORS (L. T.), « The use of atropine and adrenaline in quinuronium sulphate poisoning of cattle », Vet, Rec., 1966, 79: 771772.

CERNAIANU (C.), GLUHOVSCHI (N.) et RADEF (J.), «Acaprin, ein neues Heilmittel für Piroplasmosen *, Z. Infekt Kr. Haustiere, 1935, $48: 185-195$.

CERNAIANU (G.), RADEFF (I.) et RADESCU (T.), «III. L'acaprine dans le traitement de la piroplasmose vraie des bovidés due à Piroplasma bigeminum (Smith et Kilborne) », Bull. Soc. Path. exot., 1935, 28 : 804-806.

CERRUTI (C. G.) et FANTONI (F.), « Ricerbe sulla chemioterapia della piroplasmosi con il nuovo prodotto Bayer "Acaprin " ", Clin. vet, Milano, 1935, $\mathbf{5 8}$ : 542-552.

CURASSON (G.), «Traité de protozoologie vétérinaire et comparée. Tome III. Sporozoaires", Paris, Vigot Frères, 1943, 493 p.
DONATIEN (A.) et LESTOQUARD (F.), « Sur l'emploi du trypanbleu dans le traitement des piroplasmoses des ruminants », Bull. Soc. Paih. exot., 1927, 20 : 64-77.

GARNHAM (P. C. C.), « Malaria parasites and other Haemosporidia \&, Oxford, Blackwell Scientific Publications, 1966, $1114 \mathrm{p}$.

GILBER et AVELANGE, "La diamidine dans le traitement de la piroplasmose du cheval», Rec. Méd. vét., 1947, 123: 226-229 (Vet. Bull., 1948, $18: 348)$.

JANSEN (B. C). "The parasiticidal effect of Aureomycin (Lederle) on Babesia equi (Laveran 1899) in splenectomised donkeys », Onderstepoort $J$. vet. Res., 1953, $26: 175-182$.

JOHNSTON (L. A. Y.) et CALLOW (L. L.), « Intracerebral inoculation and brain biopsy in cattle», Aust, vet. J., 1963, 39 : 22-24.

KEMRON (A.), PIPANO (E.), HADANI (A) et NEUMAN (M.), « Trials with a diamidine compound (M \& B 5062A) in the treatment of Babesiella berbera infection in cattle ", Refuah vet., 1960, 17: 236-226.

LOURIE (E. M.) et YORKE (W.), « Studies in chemotherapy. XXII. - The action of certain aromatic diamidines on Babesia canis infections of puppies », Ann. trop. Med. Parasit., 1939, 33: 305-312.

MILLER (J.G.), «The prevention and treatment of anaplasmosis », Ann. N. Y. Acad. Sci., 1956, 64: 49-55.

NUTTALL (G. H. F.) et HADWEN (S.), «The succesful drug treatment of canine piroplasmosis, together with observations upon the effect of drugs on Piroplasma canis », Parasito!ogy, 1909, $2: 156-191$.

PANISSET (L.) et GRASSET (E.), «Sur l'emploi thérapeutique des matières colorantes. Quelques essais avec un composé de l'acridine, la Gonacrine (chlorométhylate de diamino-acridine). Fixation par les tissus. Elimination par la mamelle », Bull. Acad. vét. France, 1931, 4: 131-143.

PIPANO (E.). "The effect of treatment with amicarbalide isethionate (Diampron, May and Baker) on premunition against Babesia bigemina in splenectomized cattle ", J. Prot., 1966, 13 (suppl. août) : 35 . 
RAMPON (L.), « La gonacrine dans le traitement des piroplasmoses algériennes ", Bull. Soc. Path. exot., 1933, 26 : 1002-1004.

RAYNAÚD (J.P.), « Morphologie, chimiosensubilité et réactions immunitaires de souches de Babesia bigemina (Smith et Kilborne, 1893) muses en évidence par splénectomie de bovins», Rev. Elev. Méd. vét. Pays trop., 1962, 15: 167-179.

RIEK (R. F.), «Babesiosis. In: WEINMAN D. et RISTIC M. Infectious blood diseases of man and animals. Vol. II. The pathogens, the infections and the consequences », New York, London, Academic Press, 1968, p. 219-268.

SEIFERT (H.), "Die Bekämpfung eines "Rindersterbens " in der Cordillere Nord-Perus», $Z b l$. Vet. Med., 1960, 7: 991-1015.

SERGENT (E.), DONATIEN (A.), PARROT (L.) et LESTOQUARD (F.), «Etudes sur les piroplasmes bovines ", Alger. Institut Pasteur d'Algérie 1945, 816 p.

SHONE (D. K.), WELLS (G. E.) et WALLER (F.J.A.), «The activity of Amicarbalide against Babesia bigemina ", Vet. Rec., 1961, 73: 736-740.

STEPHAN (O.) et ESQUIBEL (A.), "Methodo de premunição contra a "Trısteza " usado no posto zootechnico de Săo Paulo », Arch. Inst. Biol. Def. Agric. Anim., São Paulo, 1929, 2 : 183-208.

TCHERNOMORETZ (I.), «Blocking of the brain capillaries by parasitized red blood-cells in Babe- sia berbera infections in cattle », Ann. trop. Med. Parast., 1943, 37 : 77-79.

UILENBERG (G.). «Sur la pathogénie des formes cérébrales des babésioses bovines à Madagascar », Rev. Elev. Méd. vét. Pays trop., 1965, 18: 83-88.

UILENBERG (G.), « Note sur la piroplasmose équine à Madagascar », Rev. Elev. Méd. vét. pays trop., 1976, 20 : 497-500.

UILENBERG (G.), « Notes sur les babésioses et l'anaplasmose des bovins à Madagascar. I. - Introduction. Transmission », Rev. Elev. Méd. vét. Pays trop., 1968, 21 : 467-474.

UILENBERG (G.), « Notes sur les babésioses et l'anaplasmose des bovins à Madagascar. II. - Influence de la splénectomie», Rev. Elev. Méd. vét. Pays trop., 1969, 22 : 237-48.

UILENBERG (G.), "Notes sur les babésioses et l'anaplasmose des bovins à Madagascar. IV. - Note additionnelle sur la transmission", Rev. Elev. Méd. vét Pays trop. (sous presse).

VOGELSANG (E. G.) et PIERETTI (R V), «Experiencas con el spirotripan "fuerte" en el tratamiento de la anaplasmosis bovina ", Rev. Med. vet., Caracas, 1957, 16: 71-77 (Vet. Bull., 1959, $29: 182$ ).

ZUBIATE (A), "Behandlung der Anaplasmose der Rinder mit Spirotrypan "forte" ad us. vet. ", Vet.-med. Nachr., 1956 (3) : 181-184. 